\title{
Gyrokinetic analysis of low- $n$ shear Alfvén and ion sound wave spectra in a high-beta tokamak plasma
}

\author{
Andreas Bierwage ${ }^{1}$ and Philipp Lauber ${ }^{2}$ \\ 1 National Institutes for Quantum and Radiological Science and Technology, \\ Rokkasho Fusion Institute, Aomori 039-3212, Japan \\ 2 Max Planck Institute for Plasma Physics, EURATOM Association, D-85748 \\ Garching, Germany \\ E-mail: bierwage.andreas@qst.go.jp
}

\begin{abstract}
Using the linear gyrokinetic code LIGKA, we study the structure of the continuous spectra $\Omega(\rho)=\omega(\rho)+i \gamma(\rho)$ of shear Alfvén waves (SAW) and ion sound waves (ISW) in a high-beta JT-60U tokamak plasma and look for evidence of Alfvén acoustic couplings or mode conversion. Here, $\Omega$ is the complex local eigenfrequency, $\rho$ is a radial coordinate, and we consider waves with low toroidal mode number $n=3$. We focus on the frequency range $\omega_{\mathrm{BAE}} \lesssim \omega \lesssim \omega_{\mathrm{TAE}}$ between the beta-induced and toroidicity-induced Alfvén frequency gaps. The real frequencies $\omega(\rho)$ of the gyrokinetic ISW continua are remarkably similar to MHD results. The kinetic damping rates are of order $-\gamma / \omega \sim 30 \%$ for $T_{\mathrm{e}} / T_{\mathrm{i}} \approx 1.7$, and reduce to $15 \%$ when the temperature ratio is raised to $T_{\mathrm{e}} / T_{\mathrm{i}} \approx 4$.8. It is shown that SAW and ISW continua can be simultaneously excited with an antenna and that the global response of the ISWs is significantly enhanced when the on-axis beta value is raised from $\beta_{0}=1.7 \%$ to $3.6 \%$ while keeping $T_{\mathrm{e}} / T_{\mathrm{i}}>1$. In contrast, when the ion temperature is increased such that $T_{\mathrm{e}} / T_{\mathrm{i}} \approx 0.4$, ISW branches become undetectable in spite of higher $\beta_{0}$. At the same time, a large part of the SAW continuum is locally destabilized by ion temperature gradients (ITG) and a set of discrete global modes was found, some of which are weakly damped or unstable and interpreted as kinetic beta-induced Alfvén eigenmodes (KBAE). It is estimated that the kinetic damping of such low- $n$ Alfvénic modes contributes much more to the anomalous bulk ion heating than the excitation of nearby ISW continua, so that Alfvén acoustic couplings in the frequency band $\omega_{\mathrm{BAE}} \lesssim \omega \lesssim \omega_{\mathrm{TAE}}$ are probably irrelevant in practice.
\end{abstract}

\section{Introduction}

\subsection{Motivation and goal}

The structure of the continuous spectra of magnetohydrodynamic (MHD) waves in magnetic confinement devices contains information that is valuable for various purposes, such as MHD spectroscopy and the identification of frequency gaps, discrete modes and possible resonances with fast ions. Moreover, if one takes into account wave damping mechanisms, one may use the continuous spectra to study channels via which energy can be transferred between plasma gradients, collective oscillations and heat. 
In the present work, we are interested in the properties of and couplings between kinetic Alfvén waves (KAW) and ion sound waves (ISW) in the core of high-temperature tokamak plasma as used in nuclear fusion research. Such plasmas are characterized by relatively strong nonuniformities (i.e., spatial and velocity gradients) as well as low collision rates. The nonuniformities give rise to a large variety of discrete modes and continuous spectra with a complicated structure. The low collisionality implies that the decay rates of global wave packets are primarily determined by phase mixing processes (namely, continuum damping and Landau damping), before the resulting small-scale structures are dissipated by collisional drag and diffusion.

Although the importance of diamagnetic effects and kinetic compression is wellrecognized, the continuous spectra of KAWs and SAWs are often computed using the standard single-fluid full MHD equations. This means that the KAW and ISW branches are idealized, respectively, by ideal shear Alfvén waves (SAW) and slow magnetosonic ("MHD sound") waves, which propagate along the magnetic field.

In the present study, we compare MHD wave spectra with those of a gyrokinetic (GK) model under realistic fusion-relevant tokamak conditions, where the plasma is strongly shaped and has a beta value well above $1 \%$ (ratio of thermal to magnetic pressure). The ratio of electron to ion temperature, which depends on the plasma heating scheme and has a strong effect on ISW damping, will be used as a free parameter and varied in the range $0.4 \lesssim T_{\mathrm{e}} / T_{\mathrm{i}} \lesssim 4.8$.

One purpose of this work is to determine whether MHD sound continua can serve as a convenient albeit approximate measure to classify modes seen in experiments or GK simulations. The second purpose is to examine whether couplings or mode conversions between SAWs and MHD sound waves that were seen in recent global MHD simulations [1] have a counterpart in the GK description. We will then discuss implications for anomalous heating of bulk ions via Alfvén acoustic couplings as proposed in Ref. [1] and the role of fast ions. As a preparation, the basic concepts and some related previous works are reviewed in the following sections.

\subsection{Review: Continuous and discrete spectra of MHD waves}

If one ignores geometric couplings between different poloidal harmonics for simplicity, the dispersion relations determining the angular frequencies $\omega_{\mathrm{A}}$ and $\omega_{\mathrm{S}}$ for SAW ("A") and MHD sound waves ("S") can be written as

$$
\omega_{\mathrm{A}}^{2} \approx k_{\|}^{2} v_{\mathrm{A}}^{2}, \quad \omega_{\mathrm{S}}^{2} \approx k_{\|}^{2} v_{\mathrm{S}}^{2} .
$$

The Alfvén and MHD sound velocities $v_{\mathrm{A}}(\rho)$ and $v_{\mathrm{S}}(\rho)$ are functions of the normalized poloidal flux $0 \leq \psi \leq 1$, whose square root $\rho \equiv \sqrt{\psi}$ will serve us as a radial coordinate from the plasma center $(\rho=0)$ to the edge $(\rho=1)$. The quantity $k_{\|}(\rho)$ is the wave number parallel to the unperturbed equilibrium ("eq") magnetic field $\boldsymbol{B}_{\text {eq }}=B_{\text {eq }} \boldsymbol{b}$, and it is defined via the Fourier transform $\boldsymbol{b} \cdot \boldsymbol{\nabla} \delta \phi(\rho, \vartheta, \zeta) \leftrightarrow i k_{\|}(\rho) \delta \phi_{m, n}(\rho)$ as

$$
k_{\|}(\rho) \equiv \frac{1}{R_{0}}\left(n-\frac{m^{ \pm}}{q(\rho)}\right) \gtrless 0 ;
$$



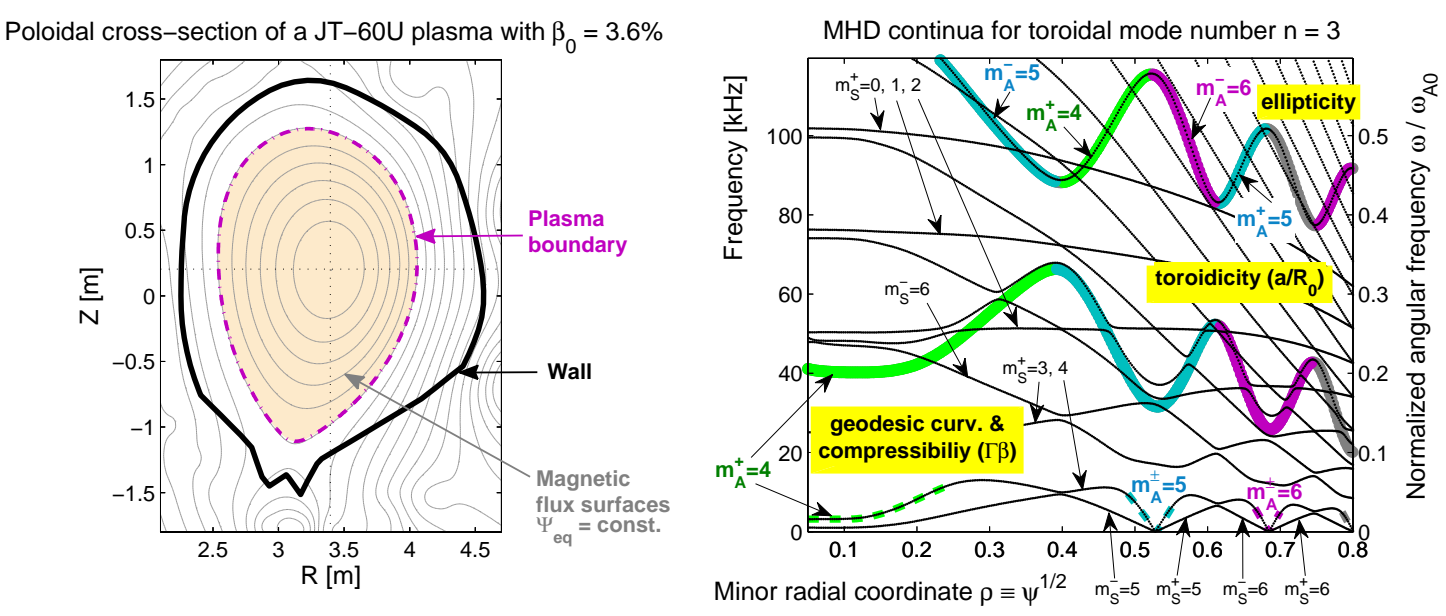

Figure 1. Poloidal cross-section of the equilibrium magnetic flux surfaces $\Psi_{\text {eq }}=$ const. in a strongly shaped high-beta JT-60U tokamak plasma (left) and the structure of its MHD wave continua (right). This deuterium plasma has a central toroidal beta value of $\beta_{0} \approx 3.6 \%$ and inverse aspect ratio $a / R_{0} \approx 0.3$. The right panel shows the full MHD continua (thin black) and the SAW continua in the slow-sound approximation (bold colored) for toroidal mode number $n=3$ and specific heat ratio $\Gamma=5 / 3$. The poloidal mode numbers of several shear Alfvén $\left(m_{\mathrm{A}}\right)$ and MHD sound $\left(m_{\mathrm{S}}\right)$ branches are indicated.

where $q(\rho)$ is the safety factor, and $m$ and $n$ are the wave numbers of the perturbed electrostatic (ES) potential $\delta \phi(\rho, \vartheta, \zeta)$ along the poloidal and toroidal angles $\vartheta$ and $\zeta$. As in Ref. [1], the superscripts on the poloidal mode number $m^{ \pm}$identify the sign of $k_{\|}$.

In realistic plasma geometry, the radial structure $\omega(\rho)$ of the MHD continua is more complicated than in Eqs. (1) and (2) because of geometric couplings and effects of finite compressibility. A good approximation of the SAW continua $\omega_{\mathrm{A}}(\rho)$ can be obtained by numerically solving the full set of MHD equations in the so-called "slow sound approximation" [2]. As an example, the colored bold lines in Fig. 1 show the SAW continua $\omega_{\mathrm{A}}(\rho)$ for fluctuations with toroidal mode number $n=3$ in a high-beta JT-60U tokamak plasma consisting primarily of fully ionized deuterium.

Below $40 \mathrm{kHz}$, one can see the so-called "beta-induced" shear Alfvén frequency gaps [2], which arise from the combined effects of the geodesic curvature of the magnetic field and ion compressibility in a finite-beta plasma. The role of curvature is to couple the pressure fluctuations with the SAW dynamics. Given this coupling, the beta-induced gaps exist because incompressible SAWs can propagate in a compressible plasma only when their frequency exceeds a certain threshold. The accumulation points (extrema) at the bottom of the SAW continua give rise to so-called beta-induced Alfvén eigenmodes (BAE) $[3,4]$, so one often speaks of "BAE gaps" for non-zero toroidal mode numbers $n>0$. For $n=0$, the beta-induced gap is bounded by the continuum of geodesic acoustic modes (GAM) [5], so one may also say that the BAE gap represents the upshift of the SAW continua by the local GAM frequency. 
Around $80 \mathrm{kHz}$, one can see the toroidicity-induced shear Alfvén gaps, which arise from the linear toroidal coupling between neighboring SAW harmonics $(|\Delta m|=1)$ with opposite signs of $k_{\|}$. Under certain conditions, there exist discrete standing waves inside these gaps. These are called toroidicity-induced Alfvén eigenmodes (TAE) [6, 7, 8], so one often speaks of "TAE gaps". At higher frequencies, gaps along with corresponding Alfvén eigenmodes exist due to couplings facilitated by higher-order plasma shape harmonics, such as ellipticity $(|\Delta m|=2$, here around $130 \mathrm{kHz})$, triangularity $(|\Delta m|=3$, here around $200 \mathrm{kHz}$ ), and so forth.

Without the slow-sound approximation one obtains the full MHD spectra shown as thin black lines in Fig. 1, which contain both the SAW and the MHD sound branches. These continua were computed by applying the numerical method described in Ref. [9] to the standard single-fluid full MHD equations, where the MHD sound velocity is

$$
v_{\mathrm{S}}^{2}=\Gamma P_{\mathrm{bulk}} /\left(m_{\mathrm{i}} n_{\mathrm{i}}\right) .
$$

Here, $P_{\text {bulk }}$ is the thermal pressure of the bulk plasma, $m_{\mathrm{i}} n_{\mathrm{i}}$ is the ion mass density, and the value of the specific heat ratio is chosen to be $\Gamma=5 / 3$.

Similarly to the SAW branches discussed above, the different poloidal harmonics $m_{\mathrm{S}}^{ \pm}$ of MHD sound waves are also subject to mutual geometric couplings due to torodicity and plasma shaping. In addition, geodesic curvature facilitates coupling between MHD sound waves and low-frequency branches of SAWs that are modified by finite plasma compressibility. Here, the latter will be referred to as "compressible SAWs" and they are highlighted in Fig. 1 by bold dashed lines around $10 \mathrm{kHz}$ and below. The frequency gaps produced by geodesic Alfvén acoustic couplings between neighboring harmonics $m_{\mathrm{A}}$ and $m_{\mathrm{S}}=m_{\mathrm{A}} \pm 1$ can be seen in Fig. 1 around $5-20 \mathrm{kHz}$. The associated accumulation points give rise to so-called beta-induced Alfvén acoustic eigenmodes (BAAE) [10, 11, 12], so one often speaks the "BAAE gaps".

In MHD, higher-order Alfvén acoustic gaps can also be found at higher frequencies. In the example shown in Fig. 1, couplings up to third order $|\Delta m|=3$ (for instance at $60 \mathrm{kHz}$ near $\rho \approx 0.3$ ) can be clearly seen due to the relatively large triangularity of the magnetic surfaces.

\subsection{Review: Relation between MHD and GK descriptions}

It is important to note that the geometric couplings between SAW and MHD sound wave branches with $\left|m_{\mathrm{A}}-m_{\mathrm{S}}\right|>1$ in Fig. 1 are a consequence of the fact that the linearized ideal MHD model yields only real eigenfrequencies, which means that pure MHD waves are not subject to any form of local damping. $\ddagger$ The spatio-temporal interference between two different wave branches 1 and 2 can produce a robust gap around radii where $\omega_{1} \approx \omega_{2}$ only if their growth or damping rates are similar, such that

$$
\left|\gamma_{1}-\gamma_{2}\right| / \omega \ll 1
$$

$\ddagger$ The so-called "continuum damping" refers only to the loss of coherence in a global wave packet; i.e., the rate at which the envelope of a global wave packet decays due to the radial variation of the local frequencies, $\mathrm{d} \omega_{\mathrm{A}} / \mathrm{d} \rho$ and $\mathrm{d} \omega_{\mathrm{S}} / \mathrm{d} \rho$. 
In other words, there must be sufficient time for establishing the parallel waveform with well-defined $k_{\|}$before the individual amplitudes of the interfering wave branches deviate too much. For SAWs and ISWs in the gyrokinetic (GK) description, the condition in Eq. (4) is usually violated at frequencies near and above the beta-induced gap, $\omega \gtrsim \omega_{\mathrm{BAE}}$, where the SAW and ISW branches tend to have very different damping rates $-\gamma_{\mathrm{S}} / \omega_{\mathrm{S}} \gg-\gamma_{\mathrm{A}} / \omega_{\mathrm{A}}$. This is because of the electron-ion temperature ratio in tokamaks is usually $T_{\mathrm{e}} / T_{\mathrm{i}} \sim 1$, which leads to strong ion Landau damping of ISWs. For the JT-60U scenario studied in the present paper, we will show that the kinetic damping rates of SAWs and ISWs differ by two orders of magnitude, so that no Alfvén acoustic gaps can form for branches with $\omega \gtrsim \omega_{\mathrm{BAE}}$, where $\left|m_{\mathrm{A}}-m_{\mathrm{S}}\right|>1$.

Significant differences between MHD and GK results, at least quantitatively, are also expected to be found for wave spectra in the low-frequency range $\omega \lesssim \omega_{\mathrm{BAE}}$, because in our high-beta JT-60U scenario (with $\beta>1 \%$ ) the ion diamagnetic frequency and ion transit frequency are not much smaller than the BAE frequency, so that

$\omega_{* \text { pi }} \lesssim \omega_{\text {ti }} \lesssim \omega_{\text {BAE }}$. This means that solutions with mixed or ambiguous polarizations may arise [13]. One may then say that the continua are shifted in the complex plane by the combined effects of diamagnetic drifts (pressure gradients), ion temperature gradient (ITG), as well as resonant and nonresonant compression of thermal ions. Therefore, the frequency range $\omega \lesssim \omega_{\mathrm{BAE}}$ may more accurately be referred to as a "kinetic thermal ion (KTI) gap" [14]. Note that the kinetic compression is also influenced by the partition of the plasma pressure among different particle species; namely, electrons, thermal ions, fast ions and other impurities. For the SAW continua, this means that the width of the BAE (or KTI) gap is likely to be different between MHD and GK descriptions, and we will show that this is also the case for the JT-60U scenario considered here.

Apart from these differences, the structure of the SAW continua predicted by MHD has been qualitatively verified; for instance, by the linear GK code LIGKA [15]. Less attention has previously been paid to the question of how well MHD sound continua correspond to the continuous spectra of ISW in the GK picture, especially for the frequency range $\omega_{\mathrm{BAE}} \lesssim \omega \lesssim \omega_{\mathrm{TAE}}$, which (according to our knowledge) is addressed here for the first time.

\subsection{Review: Alfvén acoustic energy channel in the BAE-TAE frequency range}

The frequency band $\omega_{\mathrm{BAE}} \lesssim \omega \lesssim \omega_{\mathrm{TAE}}$ has attracted our interest due to observations of chirping energetic particle modes (EPM [16]) and abrupt large events (ALE) in beam-driven JT-60U experiments [17], which were then intensively studied numerically using MHD-kinetic hybrid codes [18, 19, 20] (and references therein). Moreover, just in that frequency range, recent results of MHD simulations yielded evidence for Alfvén acoustic couplings [1], which give rise to so-called beta-induced Alfvén continuum modes (BACM), as shown in Fig. 2. In this example, which is based on a beam-driven JT60U tokamak plasma with a high central toroidal beta of $\beta_{0}=3.6 \%$, fluctuations with toroidal mode number $n=3$ were examined, and the couplings occurred between SAWs 


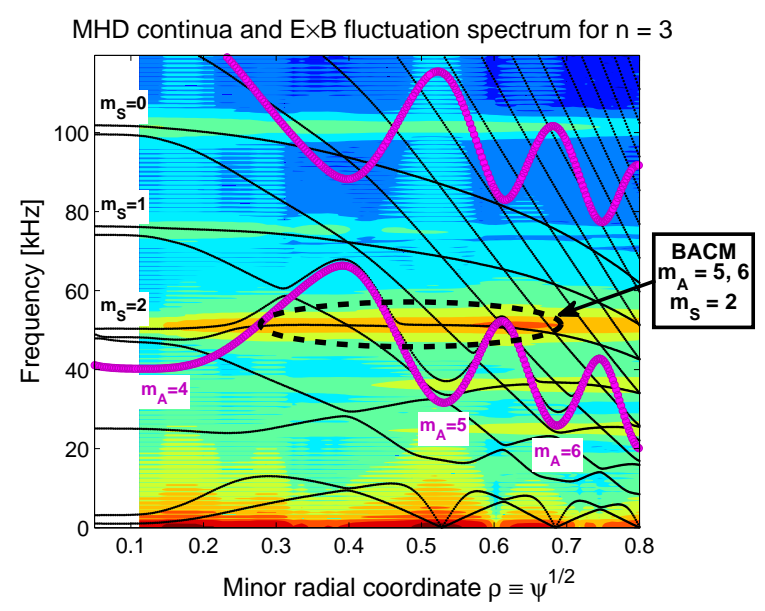

Figure 2. Beta-induced Alfvén continuum modes (BACM) with toroidal mode number $n=3$, as found in simulations of the MHD response of a high-beta JT-60U tokamak plasma [1]. The log-scale color contours represent the amplitude of the radial $\boldsymbol{E} \times \boldsymbol{B}$ velocity component $\delta u_{r}$ in the frequency-radius plane. Full MHD continua (thin black) and shear Alfvén continua (bold magenta) from Fig. 1 are superimposed for comparison. The BACM resulting from the coupling between the poloidal harmonics $m_{\mathrm{A}}=5$ and 6 of the shear Alfvén branch with the $m_{\mathrm{S}}=2$ harmonic of the MHD sound branch is encircled with a dashed line. See Ref. [1] for further details. The data plotted here were shown in a somewhat different form in Fig. 2 of Ref. [1]. In the present paper, the plasma response for this case is studied with a GK model.

with poloidal harmonic $m_{\mathrm{A}}=4-6$ and MHD sound waves with $m_{\mathrm{S}}=1-2$.

In this paper, we will demonstrate that similar global excitations of ISW continua in the frequency band $\omega_{\mathrm{BAE}} \lesssim \omega \lesssim \omega_{\mathrm{TAE}}$ can be found in the GK model driven by an antenna, in spite of the fact that the largely different damping rates $\left(-\gamma_{\mathrm{A}} \ll-\gamma_{\mathrm{S}}\right)$ prevent geometric couplings between SAW and ISW continua. The measured fluctuation amplitudes are then used to estimate the relative importance of Alfvén acoustic couplings as an energy channel for anomalous heating of thermal ions.

\subsection{Outline}

The contents of this paper are as follows. In Section 2, we describe the simulation scenario based on a JT-60U tokamak plasma, revisit the model equations solved by LIGKA, and provide an itemized list of current limitations and simplifying assumptions.

Section 3 is dedicated to comparisons between the single-fluid full MHD continua and the kinetic continua computed by LIGKA in the local limit [15]. Previous LIGKA studies have looked only at unstable and weakly damped solution of the GK dispersion relation. Thus, ISW continua of long-wavelength modes were inspected only in the domain of very low frequencies $\omega<\omega_{\mathrm{BAAE}}[15,21]$. In the present study, the Nyquist contour integration [22] in LIGKA is performed with about 1000 times higher resolution than usual, so that roots of the dispersion relation can be detected even when the complex frequency plane $\Omega=\omega+i \gamma$ is scanned all the way into the strongly damped 
domain, with ratios of growth rates $\gamma$ to angular frequencies $\omega$ ranging as far as $-70 \% \lesssim \gamma / \omega \lesssim 70 \%$. In this way, broad-band ISW continua are successfully computed using LIGKA for the first time. Cases with electron-ion temperature ratios in the range $0.4 \lesssim T_{\mathrm{e}} / T_{\mathrm{i}} \lesssim 4.8$ are then analyzed.

In Section 4, we examine the global GK plasma response using LIGKA's kinetic antenna model $[21,23]$ and a vector iteration method [15]. The temperature ratio $T_{\mathrm{e}} / T_{\mathrm{i}}$ and plasma beta are varied systematically, in order to test the responsiveness of the ISW continua. The results and possible implications for anomalous bulk ion heating are discussed in Section 5, and a summary and conclusions are given in Section 6 .

Note that some of the results presented here are similar to those reported at the 14th IAEA Technical Meeting on Energetic Particles in Magnetic Confinement Systems [24]. Some minor quantitative changes are due to a more self-consistent treatment of sideband couplings in the quasineutrality condition. Moreover, as additional results became available, some of the interpretations presented here differ from those given in the proceedings paper [24].

\section{Model and methods}

\subsection{Simulation scenario}

The simulation scenario is based on a high-beta JT-60U tokamak plasma (shot E039672 at 4 seconds [25]) that was driven by a pair of tangentially injected negative-ion-based neutral beams (N-NB). In this work, we focus on the toroidal harmonic $n=3$, which has been intensively studied using MHD and hybrid simulation in Refs. [1, 20, 26, 27, 28, 29]. The simulation setup was described in detail in Ref. [26]. The radial profiles of plasma parameters used in the gyrokinetic (GK) simulations are shown in Fig. 3. The ratios of thermal to Alfvén velocities in the reference case are $v_{\mathrm{te} 0} / v_{\mathrm{A} 0} \approx 6.2$ and $v_{\mathrm{ti} 0} / v_{\mathrm{A} 0} \approx 0.08$, where we use the definition $v_{\mathrm{t} a} \equiv \sqrt{2 T_{a} / m_{a}}$. The subscript $a=\mathrm{e}, \mathrm{i}, \ldots$ identifies the particle species (here, bulk electrons "e" and ions "i") and " 0 " refers to the value at the magnetic axis. The energy of the fast ("f") N-NB ions in JT-60U was as high as $E_{\mathrm{f}}=400 \mathrm{keV}$, which corresponds to a super-Alfvénic velocity ratio of $v_{\mathrm{f} 0} / v_{\mathrm{A} 0}=1.432$.

Effects of fast ions will be studied in a separate paper and are ignored here. Impurities (mostly carbon) are also ignored. Consequently, the ion number density in Fig. 3(b) will be equated to that of the electrons, $n_{\mathrm{i}}=n_{\mathrm{e}}$, in order to satisfy the quasi-neutrality constraint. The electron and ion temperatures $T_{\mathrm{e}}$ and $T_{\mathrm{i}}$ in Fig. 3(c) will be multiplied by arbitrary scaling factors in order to examine their influence on the continuous spectra and mode stability. Although this will also change the $\beta(\rho)$ profile in Fig. 3(d), we will always fix the equilibrium ("eq") magnetic flux surface geometry $\Psi_{\text {eq }}(R, Z)$ in Fig. 1 and the safety factor profile $q(\rho)$ in Fig. 3(a). 

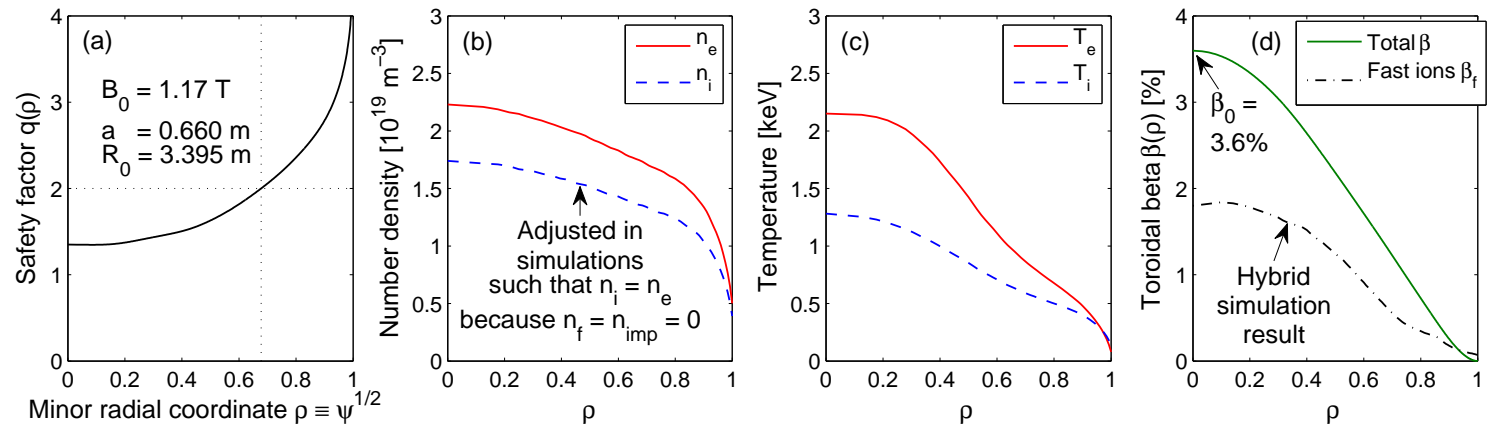

Figure 3. Plasma profiles for the reference scenario used in this work, which is based on an N-NB-driven high-beta JT-60U tokamak plasma (shot E039672 at 4 seconds [25]). Panels (a)-(d) show the radial profiles of the safety factor $q(\rho)$, the number densities $n(\rho)$ and temperatures $T(\rho)$ of thermal bulk electrons ("e") and ions ("i"), the total toroidal beta $\beta(\rho)=2 \mu_{0} P / B_{0}^{2}$ of the entire plasma and that of the fast ion component ("f"). The bulk plasma profiles are based on experimental measurements. The fast ion beta profile $\beta_{\mathrm{f}}(\rho)$ in (d) is based on the result of a long-time hybrid simulation including realistic N-NB ion sources, collisions and MHD activity [20,30]. In this work, we ignore impurities and fast ions, so that $n_{\mathrm{f}}=n_{\mathrm{imp}}=0$ and $n_{\mathrm{i}}=n_{\mathrm{e}}$.

\subsection{Gyrokinetic model}

The results presented in this paper were obtained with the code LIGKA [15, 31], which describes all particle species $a=\mathrm{e}, \mathrm{i}, \ldots$ using a linear GK model. The equations solved by LIGKA are based on the quasi-neutrality condition $0=\sum_{a} e_{a} n_{a}$ and parallel Ampère's law $[\nabla \times(\nabla \times \boldsymbol{A})]_{\|}=\mu_{0} j_{\|}$, where $\|$refers to the direction along the equilibrium component of the magnetic field $\boldsymbol{B}=\boldsymbol{B}_{\text {eq }}+\delta \boldsymbol{B}(t)$. Evaluating the fluctuations of the number densities $\delta n_{a}$ and current densities $\delta j_{a}$ in terms of the fluctuating components of the guiding center distribution functions $f_{a}=f_{\text {eq }, a}+\delta f_{a}$, we obtain the linear GK quasi-neutrality condition

$$
0=\underbrace{\sum_{a} e_{a} \int \mathrm{d}^{2} v\left\{J_{0} \delta f\right\}_{a}}_{\text {Charge density compression }}+\underbrace{m_{\mathrm{i}} \boldsymbol{\nabla}_{\perp} \cdot \frac{n_{\mathrm{i}} \boldsymbol{\nabla}_{\perp} \delta \phi}{B^{2}}+\frac{3 P_{\mathrm{i} \perp}}{4 B^{2} \Omega_{\mathrm{i}}^{2}} \nabla_{\perp}^{4} \delta \Phi}_{\text {FLR polarization terms }},
$$

and, from parallel Ampère's law, the linear GK momentum equation

$$
\begin{array}{r}
\overbrace{-\frac{\partial}{\partial t}\left[\boldsymbol{\nabla} \cdot \frac{\boldsymbol{\nabla}_{\perp} \delta \phi}{v_{\mathrm{A}}^{2}}\right]}^{\text {Inertia (2nd order) }}+\overbrace{\boldsymbol{B} \cdot \boldsymbol{\nabla} \frac{\boldsymbol{\nabla} \times\left(\boldsymbol{\nabla} \times \delta A_{\|} \boldsymbol{b}\right)}{B}}^{\text {Field line bending }}+\overbrace{\underbrace{\left(\boldsymbol{b} \times \boldsymbol{\nabla} \delta A_{\|}\right) \cdot \boldsymbol{\nabla} \frac{\mu_{0} j_{\|}}{B}}_{\text {Pressure-curvature coupling ("ballooning") }}}^{\text {Current gradient ("kink") }} \\
=\underbrace{-\sum_{a} \mu_{0} \int \mathrm{d}^{2} v e_{a}\left\{\boldsymbol{v}_{\mathrm{d}} \cdot \boldsymbol{\nabla} J_{0} \delta f\right\}_{a}}_{\text {Diamagnetic correction for inertia }}+\underbrace{\sum_{a}\left[\boldsymbol{b} \times \boldsymbol{\nabla}\left(\frac{\beta_{a \perp}}{2 \Omega_{a}}\right)\right] \cdot \boldsymbol{\nabla} \nabla_{\perp}^{2} \delta \phi}_{\text {4th-order inertia ("kinetic Alfvén wave") }} \\
\quad
\end{array}
$$


where $\beta_{a \perp}=2 \mu_{0} P_{a \perp} / B^{2}$ and where we have omitted the "eq" subscripts on equilibrium components. For species with an isotropic velocity distribution $\left(P_{\perp}=P_{\|}\right)$, the diamagnetic correction for inertia (second term on the right-hand side of Eq. (6)) can be compactly written in terms of the operator

$$
\hat{\omega}_{\mathrm{p} a}^{*}=\boldsymbol{b} \times \frac{\nabla\left(n_{a} T_{a}\right)}{i e_{a} B n_{a}} \cdot \nabla \quad \rightarrow \quad \omega_{* \mathrm{p} a} \equiv \frac{P_{a}^{\prime} k_{\vartheta}}{\Omega_{a} m_{a} n_{a}} .
$$

In the code, such differential operators are evaluated using the exact metrics, whereas the discussion of the results is made more intuitive by using a simplified notation, such as $P^{\prime} k_{\vartheta}$ with $k_{\vartheta} \approx-m / r$ and $P^{\prime} \equiv \mathrm{d} P / \mathrm{d} r$, where $r$ can be regarded as the volumeaveraged minor radius with $r / a \approx \rho$. In the following, we will refer to $\omega_{* \mathrm{p} a}$ in Eq. (7) when we speak of the diamagnetic frequency.

The evolution of the perturbed distribution functions $\delta f_{a}=f_{a}-f_{\text {eq }, a}$ written as

$\delta f_{a}=\delta h_{a}+\frac{\partial f_{\mathrm{eq}, a}}{\partial E} e_{a} J_{0}\left(\rho k_{\perp}\right)\left[\delta \phi-\delta \psi-\frac{v_{\|} k_{\|}}{\omega} \delta \psi\right]+\frac{\nabla f_{\mathrm{eq}, a}}{i \omega B} \cdot(\boldsymbol{b} \times \nabla) J_{0} \delta \psi$

is given by the linear gyrokinetic equation

$\frac{\partial \delta h}{\partial t}+\left(v_{\|} \boldsymbol{b}+\hat{\boldsymbol{v}}_{\mathrm{d}}\right) \cdot \boldsymbol{\nabla} \delta h=\left[\frac{\boldsymbol{b} \times \boldsymbol{\nabla} f_{\mathrm{eq}}}{e B} \cdot \boldsymbol{\nabla}-\frac{\partial f_{\mathrm{eq}}}{\partial E} \frac{\partial}{\partial t}\right] J_{0}\left[\delta \phi-\left(1-\frac{\hat{\boldsymbol{v}}_{\mathrm{d}} \cdot \boldsymbol{\nabla}}{i \omega}\right) \delta \psi\right]$.

with the drift velocity $\hat{\boldsymbol{v}}_{\mathrm{d}}=-\frac{\boldsymbol{b}}{e B} \times\left(m v_{\|}^{2}(\boldsymbol{b} \cdot \boldsymbol{\nabla}) \boldsymbol{b}+\mu \boldsymbol{\nabla} B\right)$ and the gyro-operator $J_{0}=J_{0}\left(\rho k_{\perp}\right)$. Equations (5)-(9) determine the eigenvalues and eigenfunctions for the perturbed electrostatic and magnetic potentials, $\delta \phi(\boldsymbol{x})$ and $\partial_{t} \delta A_{\|}(\boldsymbol{x})=-\boldsymbol{b} \cdot \boldsymbol{\nabla} \delta \psi(\boldsymbol{x})$. The definitions of all quantities and details about the numerical implementation can be found in Ref. [15].

\subsection{Limitations and approximations}

For the case studies and extensive scans of the complex frequency plane that were required for the present study, it is not feasible to use the so-called "full-orbit version" of LIGKA, which performs phase space integrals along orbits computed by the code HAGIS [32]. Instead, we employ the "analytical version" of LIGKA, which has been optimized for speed by replacing numerical solutions with analytical expressions wherever possible. At present, some effects have not yet been implemented. Below is a list of the current limitations and simplifying assumptions that were used in the present work:

1. The fast-circulating approximation [15] is used because analytical expressions for trapped particle effects have not been implemented yet. Their inclusion may alter the plasma response in the low-frequency domain, $\omega \sim \omega_{\mathrm{ti}} \sim \omega_{* \mathrm{pi}} \lesssim 0.1 \cdot \omega_{\mathrm{A} 0}[33,34]$.

2. Isotropic Maxwellian distributions are used for all species. This is reasonable for the thermal bulk plasma considered in this paper and allows one to speed up the calculations by using the standard plasma dispersion function $Z(\xi)$. A generalization as proposed in Ref. [35] is under consideration. 
3. The transition between the plasma dispersion function $Z(\xi)$ and its large argument expansion very close to rational surfaces $k_{\|} \approx 0$ can lead to numerical artifacts that were removed from the plots shown is this paper.

4. Finite-Larmor-radius (FLR) effects other than the diamagnetic correction for the inertia term are ignored when solving the local dispersion relation, which is then given by Eq. (20) of Ref. [15], in agreement with Ref. [36]. In the global analysis, FLR effects of all species are taken into account only up to second order in $k_{\perp} \varrho_{a}$, where $\varrho_{a}=v_{\perp} / \Omega_{a}$ is the Larmor radius. This approximation may quantitatively affect the radial structure and propagation properties of kinetic Alfvén waves (KAW) driven directly by an antenna or indirectly via coupling to antenna-driven Alfvén eigenmodes.

5. Finite-orbit-width (FOW) effects associated with magnetic drifts across flux surfaces are not included yet. A model accounting for FOW has recently been implemented and will be used to study the effect of fast ions in the future.

6. Due to the fast-circulating particle approximation (item 1) geometric coupling in the kinetic ion (and electron) response is included only up to first order (geodesic). Thus, ISWs with poloidal mode number $m$ couple only with neighboring harmonics $m \pm 1$ of SAWs or other ISWs. This can be justified as discussed in Section 1.3.

7. Electron Landau damping is [37] underestimated (effectively absent) in regions away from rational surfaces because only trapped electrons can satisfy $k_{\|} v_{\|} \sim 1$ for finite $k_{\|}$, but trapped particle motion is not included (item 1). In contrast, near rational surfaces, where circulating electrons can satisfy $k_{\|} v_{\|} \sim 1$ due to vanishing $k_{\|}$, the electron Landau damping is typically slightly overestimated due to the fastcirculating approximation (item 1).

8. The leading-order terms of the compressional magnetic response $\delta B_{\|}$(e.g., see Eq. (48) in Ref. [38]) are known to cancel exactly with the high-beta correction of the magnetic drift. Apart from this important self-consistent cancellation (which otherwise cause unphysical instabilities [39]), effects of $\delta B_{\|}$are ignored.

9. A pure Deuterium plasma is considered. Impurities (mostly Carbon) are ignored.

We expect that the results for the local and global response of the thermal bulk plasma reported in this paper are qualitatively valid and quantitatively accurate to lowest order.

\section{Local analysis results: Continuous spectra of SAW and ISW}

In this section, we analyze the local response of the thermal bulk plasma in the absence of fast ions. By ignoring all kinetic effects, it can be demonstrated (e.g., as in Ref. [40]) that the GK system discussed here contains the reduced MHD model [41] as a subset. Therefore, it is physically meaningful to compare the respective continuous spectra and identify SAW and ISW branches as we will do in this section. Namely, we analyze the differences between a simple model of a compressible ideal MHD fluid with a constant specific heat ratio $\Gamma=5 / 3$, and a more sophisticated GK model, which includes the 
kinetic responses of thermal electrons and ions, while ignoring nonlocal and polarization effects associated with FLR and FOW corrections as described in items 4 and 5 of Section 2.3 above.

In analogy to continuous spectra of MHD waves, the concept of "kinetic continua" as singular local solutions of the GK equations can be introduced in two ways:

(a) The initial-value approach describes the collapse of the radial mode structure into a singularity. This can be achieved analytically by examining the properties of the remaining first-order differential equation that one obtains after setting the second-order term to zero (as in Appendix B of Ref. [13]).

(b) The eigenvalue approach postulates that a singularity has formed at some infinite time. Here, one formally lets the width of the radial wavenumber spectrum diverge $\left(\left|k_{r} L\right| \rightarrow \infty\right.$, where $L$ is the radial scale length of the background plasma profiles) for the leading-order solution inside the inertial layer, while assuming at the same time that $k_{r} \varrho_{\mathrm{i}} \rightarrow 0$; i.e., ignoring FLR/FOW polarization effects.

In either case, one assumes that the radial structure possesses two distinct spatial scales, where the short-scale components represent the various continuous spectra. Thus, both approaches lead to a unique and physically meaningful definition of the kinetic continuum in terms of (time-asymptotically) singular plasma oscillations, as in ideal MHD. This formulation also remains valid when diamagnetic drifts (finite $k_{\vartheta} \varrho_{a}$ ) are included by renormalization of the inertia term [13]. While ideal MHD continua capture only the resonant absorption of SAW and ISW [42, 43], the kinetic continua as defined above also account for the local kinetic damping or resonant excitation of these waves (e.g., giving rise to the local limit of Alfvénic ITG modes [44]).

The numerical methods are described in Section 3.1. The continuous spectra in the reference scenario are examined without and with diamagnetic effects in Sections 3.2 and 3.3. These results are then compared to the situation with increased electron temperature $T_{\mathrm{e}}$ (Section 3.4) and increased ion temperature $T_{\mathrm{i}}$ (Section 3.5). Similarities and differences in comparison with MHD results are discussed in Section 3.6.

\subsection{Local analysis method}

As described in Ref. [15], Eqs. (5) and (6) can be solved as a local eigenvalue problem. Since we ignore FLR effects other than diamagnetic drifts in the local dispersion relation, the associated discretization of the continuous spectra is absent here. In this limit, the local continuum solutions can be obtained - as in ideal MHD — by looking for the nullspace (kernel) of the second-order operator of the combined quasi-neutrality (5) and GK momentum equation (6). It has been shown $[33,36]$ that the expression for the nullspace is a generalization of the dispersion relation derived in Ref. [13], and the connection to the continuous spectra was discussed at the beginning of Section 3 above. For each radial location $\rho$, the eigenvalues in the complex frequency plane $\Omega=\omega+i \gamma$ are determined using Nyquist contour integration. When plotted as a function of the minor radial coordinate $\rho$, the sets of points $\omega(\rho)$ form the continuous spectra of the kinetic 
plasma response. The values of the local growth rates $\gamma(\rho)$ are taken as a qualitative measure for the local damping $(\gamma<0)$ or drive $(\gamma>0)$ that a global mode with frequency $\omega_{\text {glob }}$ will experience at radii where $\omega_{\text {glob }}=\omega(\rho)$.

The poloidal harmonics in the range $1 \leq m \leq 13$ are included in the calculation. The Nyquist contour integration was performed using circular contours with radius $\delta \Omega_{\mathrm{Nyq}}=5 \times 10^{-4}$. Such a small radius is needed to find strongly damped ISWs, whereas marginally stable solutions, such as SAW continua, can be reliably obtained with much larger contour radii of about $\delta \Omega_{\mathrm{Nyq}} / \omega \sim 10 \%$. Each contour is represented by $N_{\mathrm{Nyq}}=16$ samples. Convergence tests with up to $N_{\mathrm{Nyq}}=64$ samples yielded the same physical roots. The only advantage of increasing $N_{\text {Nyq }}$ is that the solver yields fewer spurious solutions. However, most of this "noise" appears at radii where $k_{\|} \approx 0$, so it can be easily eliminated via post-processing (as will be done here).

In the following, the angular frequency $\omega$ and growth rate $\gamma$ will be normalized by the on-axis Alfvén frequency $\omega_{\mathrm{A} 0}=v_{\mathrm{A} 0} / R_{0}$ with $v_{\mathrm{A} 0}=B_{0} / \sqrt{\mu_{0} n_{\mathrm{i} 0} m_{\mathrm{i}}}$. Its value is $2 \pi / \omega_{\mathrm{A} 0} \approx 200 \mathrm{kHz}$ for the plasma parameters and profiles of the reference case shown in Fig. 3. Instead of using $\gamma$, we will measure the growth/damping rate using the quantity

$$
\gamma \tau=\ln \left|\frac{\delta \phi(t+\tau)}{\delta \phi(t)}\right| \quad \text { with } \quad \tau \equiv 2 \pi / \omega .
$$

For instance, $\gamma \tau=-1$ means that the fluctuation amplitude is reduced to $\delta \phi(t+$ $\tau) / \delta \phi(t)=\exp (\gamma \tau) \approx 1 / 2.7 \lesssim 37 \%$ during one oscillation period $\tau$. We will search for roots of the dispersion relation by scanning the complex frequency plane $\Omega=\omega+i \gamma$ in the domain $-0.1 \leq \omega \leq 0.5$ and $-4.5 \leq \gamma \tau \leq 4.5$.

\subsection{Reference scenario without diamagnetic effects}

For the purpose of illustration, we begin with results obtained without diamagnetic effects $\left(\omega_{*}\right.$ terms); i.e., pressure gradients are effectively turned off. We consider the reference scenario with the plasma profiles in Fig. 3, where the ratio of the electron and ion temperatures at the magnetic axis is $T_{\mathrm{e} 0} / T_{\mathrm{i} 0} \approx 1.7$. Since the contribution of fast ions is excluded here, the total plasma beta is reduced to about half of its original value, so we have $\beta_{0}=1.7 \% \approx \beta_{0}^{\text {ref }} / 2$ at the axis.

The results obtained with the local Nyquist analysis can be conveniently visualized as in Fig. 4. Panel (a) shows the eigenvalues in the complex $\omega-\gamma$ plane, and panel (b) shows the radial distribution of growth rates $\gamma(\rho)$. One can identify three domains that contain solutions that can be classified as (i) SAW, (ii) ISWs, and (iii) higher-order roots. The GK continuous spectra $\omega(\rho)$ of these three classes of solutions are shown in panels (c-i), (c-ii) and (c-iii) at the bottom of the figure. For comparison, the full MHD continua $\omega_{\text {mhd }}(\rho)$ are shown as gray lines. We will use this arrangement throughout this Section 3.

One can see in Fig. 4(c-i) that the real part $\omega(\rho)$ of the continuous spectra of SAWs are very similar to those of the MHD limit. Figures 4(a) and 4(b) show that the SAWs labeled (i) are marginally stable. In the vicinity of the toroidicity-induced gap, 


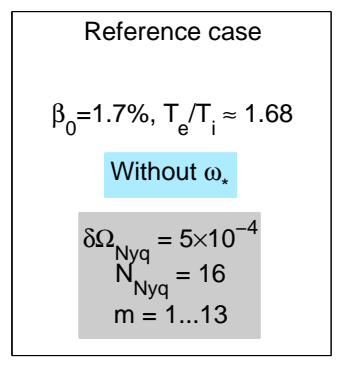

(c) Frequency spectra $\omega(\rho)$ for classes (i), (ii), (iii)
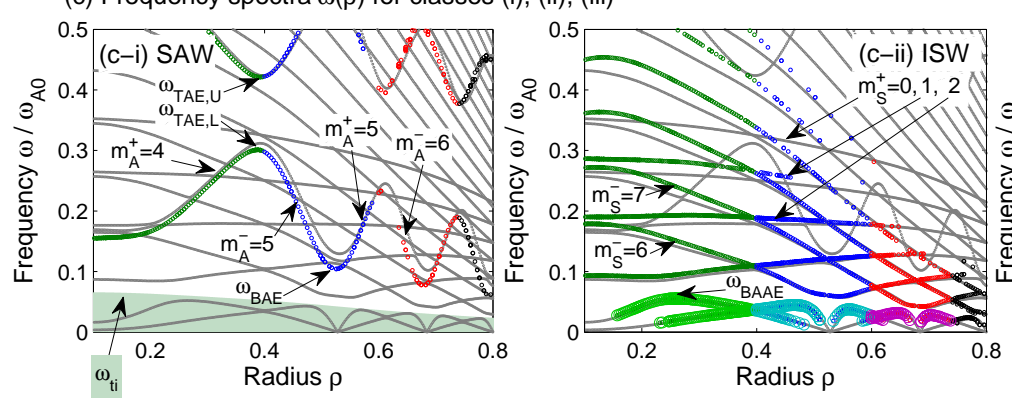
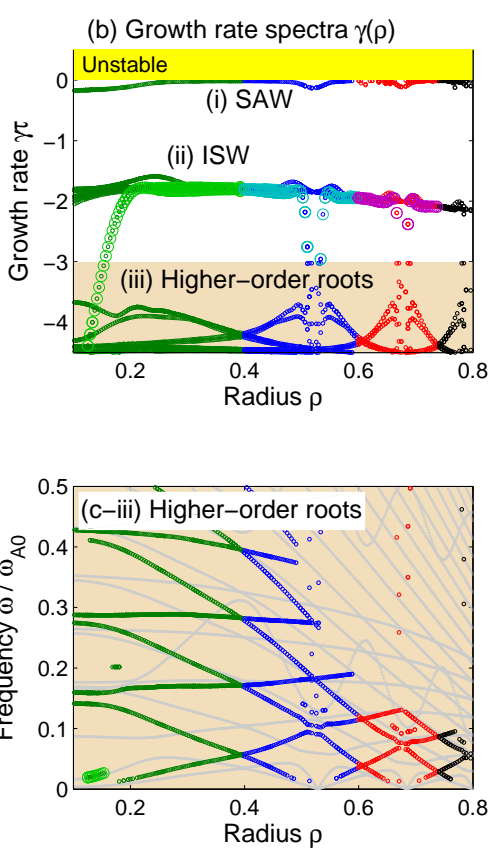

Figure 4. Results of a local analysis for the reference case with $T_{\mathrm{i}}=T_{\mathrm{i}}^{\mathrm{ref}}, T_{\mathrm{e}}=T_{\mathrm{e}}^{\mathrm{ref}}$ and $\beta_{0}=1.7 \%$ without diamagnetic effects. Panel (a) shows the eigenvalues in the complex $\omega-\gamma$ plane, and panel (b) shows the radial distribution of growth rates $\gamma(\rho)$. The solutions are classified as (i) SAWs, (ii) ISWs, and (iii) higher-order roots, which can be roughly separated by imposing thresholds on the damping rate. Here we set the thresholds at $\gamma \tau=-1$ and -3 . The corresponding continuous spectra $\omega(\rho)$ are shown in panels (c-i), (c-ii) and (c-iii). For clarity, different colors are used for eigenvalues located in four different radial intervals with boundaries at $\rho=0.4,0.6$ and 0.74 . For comparison, the MHD continua $\omega_{\text {mhd }}(\rho)$ are plotted as gray lines in the bottom panels. In panels (c-i) and (c-ii), some of the accumulation points of Alfvén acoustic and SAW spectra are labeled with $\omega_{\mathrm{BAAE}}, \omega_{\mathrm{BAE}}, \omega_{\mathrm{TAE}, \mathrm{L}}$ (lower) and $\omega_{\mathrm{TAE}, \mathrm{U}}$ (upper). The poloidal mode numbers $(m)$, the polarization $(\mathrm{A} / \mathrm{S})$, and the sign of $k_{\|}( \pm)$for some branches are also indicated. The shaded low-frequency band in panel (c-i) indicates the ion transit frequency $\omega_{\mathrm{ti}}(\rho)$.

$0.25 \lesssim \omega \lesssim 0.4$, their damping rates are around $-\gamma \tau \sim 0.02$. At lower frequencies, near the accumulation points $\omega_{\mathrm{BAE}} \sim 0.1$ of the beta-induced gap, the damping rates of SAWs increase to values of order $-\gamma \tau \sim 0.1$. As expected, the size of the beta-induced gap is proportional to the ion transit frequency $\omega_{\mathrm{ti}}(\rho)=v_{\mathrm{ti}}(\rho) /\left(q(\rho) R_{0}\right)$ with $v_{\mathrm{ti}} \equiv \sqrt{2 T_{\mathrm{i}} / m_{\mathrm{i}}}$, which is shown as a shaded region at the bottom of Fig. 4(c-i). Note, however, that $\omega_{\mathrm{BAE}} / \omega_{\mathrm{ti}} \approx 2.4$ is larger than the well-known estimate $\sqrt{7 / 4+T_{\mathrm{e}} / T_{\mathrm{i}}} \approx 1.9$.

The continua of ISWs shown in Fig. 4(c-ii) are also very similar to the results of the MHD limit; especially, in the frequency band of the beta-induced gap $(\omega \lesssim 0.1)$. Figures $4(\mathrm{a})$ and $4(\mathrm{~b})$ show that the ISWs (ii) are subject to strong damping with $-\gamma \tau \approx 2$. Low-frequency ISW branches located below the BAAE gap $\left(\omega_{\mathrm{BAAE}} \sim 0.05\right)$ are indicated by larger circles. These also include the low-frequency "compressible SAW" branches, which are subject to very strong damping $-\gamma \tau>3$ near rational surfaces and in the plasma center. 


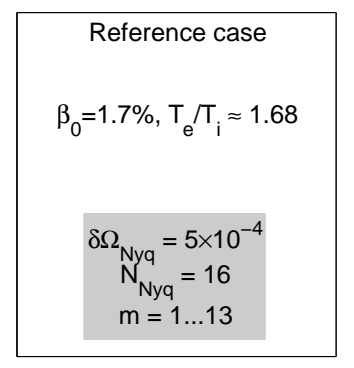

(c) Frequency spectra $\omega(\rho)$ for classes (i), (ii), (iii)
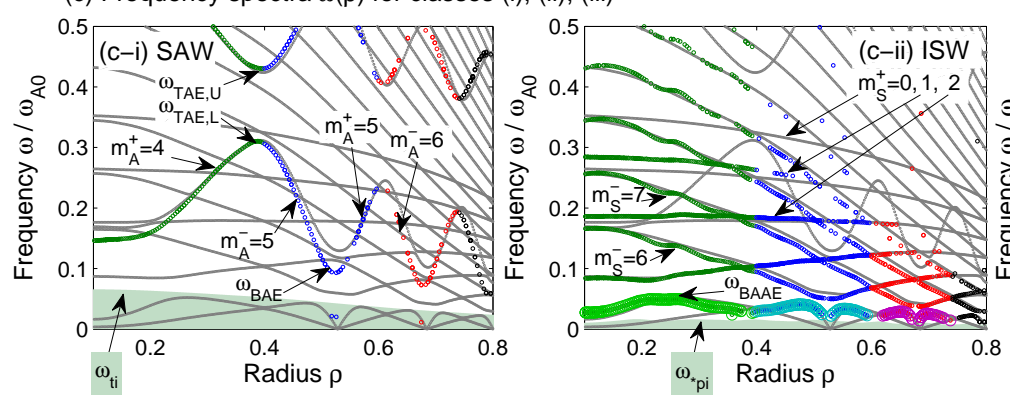
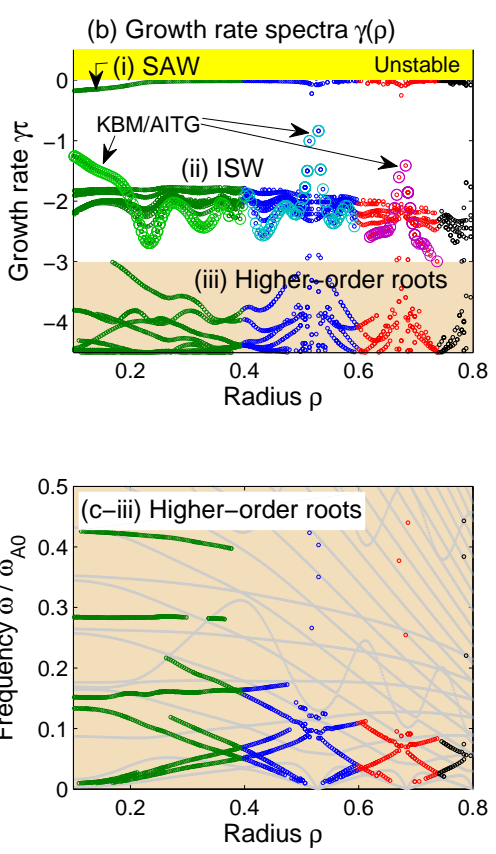

Figure 5. Local analysis results obtained in the reference case with $T_{\mathrm{i}}=T_{\mathrm{i}}^{\mathrm{ref}}$, $T_{\mathrm{e}}=T_{\mathrm{e}}^{\mathrm{ref}}$ and $\beta_{0}=1.7 \%$. The shaded low-frequency band in panel (c-ii) indicates the diamagnetic frequency $\omega_{* \mathrm{pi}}(\rho)$. Otherwise arranged as Fig. 4.

Higher-order roots labeled (iii) are also found. They have very large damping rates $-\gamma \tau \gtrsim 3.5$ and their continuous spectra are shown in Fig. 4(c-iii). These higher-order roots arise from the same term as the ISW branches. Mathematically, they can be traced back to the transcendental nature of the plasma dispersion function and are usually ignored. However, there have also been attempts to examine their possible role in other contexts.S Their physical meaning in the present case remains to be clarified.

\subsection{Reference scenario with diamagnetic effects: Destabilization of KBM/AITG}

Figure 5 shows the results for the reference case obtained with diamagnetic effects included. The profile of the ion diamagnetic frequency $\omega_{* p i}(\rho) \lesssim 0.015$ defined in Eq. (7) is shown as a shaded region at the bottom of Fig. 5(c-ii). Since the diamagnetic frequency is relatively small for the long-wavelength modes considered here $(m \sim 4-$ 7), there are only small frequency shifts in Figs. 5(c-i) and 5(c-ii), and both the SAW and ISW continua still agree with the MHD results rather well in the present case. Figures 5(a) and 5(b) show that the damping rates of both SAW (i) and ISW (ii) still lie in the domains $-\gamma \tau \lesssim 0.1$ and $-\gamma \tau \sim 2$, respectively. However, there are some differences worth noting, which are described in the following.

When comparing Fig. 5 with Fig. 4, where diamagnetic effects were ignored, one

$\mathcal{S}$ For instance, in a study of energetic-particle driven geodesic acoustic modes (EGAM) in a tokamak plasma, Girardo et al. [45] have shown that higher-order roots can be selectively excited by carefully tuning the resonant drive provided by a bump-on-tail velocity distribution. 
can see that the growth rate profiles $\gamma(\rho)$ in Fig. 5(b) are more nonuniform when $\omega_{*}$ is included. Similarly, although to a lesser degree, the spectra $\omega(\rho)$ in Fig. 5(c) have also become somewhat wiggly. These wiggles are due to variations in the radial derivatives of the experimentally measured profiles for the densities and temperatures, $n_{a}$ and $T_{a}$, which enter $\omega_{* \mathrm{p} a} \propto m\left(n_{a} T_{a}\right)^{\prime}$ with $a=\mathrm{e}$, i. Obviously, this effect increases with increasing poloidal mode number $m$. Both $\gamma(\rho)$ and $\omega(\rho)$ would be much smoother if smoother density and temperature profiles were used.

Low-frequency roots below the BAAE accumulation point, with $\omega \lesssim \omega_{\text {BAAE }} \sim 0.05$, are indicated by larger circles in Figs. 4 and Fig. 5. There, one can see the following differences between the results obtained without and with $\omega_{*}$. Firstly, the lowestfrequency portions of the ISW continua with $0<\omega \lesssim 0.02$ could not be found, although they can still be found in the negative-frequency domain $\omega<0$ (not shown here). $\|$ Secondly, one can see that "compressible SAW" roots in the plasma center and near the rational surfaces, which were strongly damped in the case without $\omega_{*}$ in Fig. 4, are destabilized in the presence of diamagnetic effects in Fig. 5. Since their frequencies are characterized by $\omega \sim \omega_{* p i}$, they may be interpreted as the local limit of kinetic ballooning modes (KBM) that are driven by ion temperature gradients (ITG). In Ref. [44], such modes were called Alfvénic ITG (AITG) modes. In Fig. 5 and in the following, we label them "KBM/AITG", where

- "KBM" identifies the wave branch — namely, low-frequency "compressible SAW" continua with $\omega<\omega_{\mathrm{BAAE}}$, which are coupled to ISW branches and modified by diamagnetic effects; $\mathcal{P}$

- "AITG" identifies the driving mechanism - namely, resonant destabilization via the pressure-curvature coupling term in the momentum equation (6), where ITG serves as an energy source. ${ }^{+}$

Let us emphasize again that the results for this low-frequency domain $\left(\omega \lesssim \omega_{\mathrm{BAAE}}\right)$ can be expected to change quantitatively when the presently ignored effects of trapped particles are taken into account. Furthermore, note that the "KBM/AITG" solutions near the plasma center $(\rho \lesssim 0.2)$ are also affected by the weak and slightly negative magnetic shear in that region. We have not analyzed the role of this effect, yet.

At higher frequencies, $\omega \gtrsim \omega_{\mathrm{BAE}}$, the effect of $\omega_{*}$ is as follows. The SAW continua in Fig. 5(c-i) are down-shifted near rational surfaces (where $k_{\|} \rightarrow 0$ ) and up-shifted elsewhere as expected [33]. The diamagnetic modification of ISW continua is more

$\|$ One possible explanation is that these low-frequency continua with $0<\omega \lesssim \omega_{* \text { pi }}$ were shifted too deep into the complex plane (strongly damped), so that the numerical accuracy of our local Nyquist analysis was insufficient to find them. In any case, a thorough study of this low-frequency domain would require to take into account trapped particle effects [33, 34].

$\mathcal{P}$ Similarly, high-frequency AITG modes appear when the usual (incompressible) SAW continua with $\omega>\omega_{\mathrm{BAE}}$ are destabilized by ITG, such as the KBAE/AITG modes discussed in Sections 3.5 and 4.2.

+ In contrast, ITG-driven electrostatic (ES) drift waves are excited via the charge density compression term in the quasi-neutrality condition (5), so that such ESITG modes tend to have lower frequencies $\omega \ll \omega_{* \text { pi }}$ (cf. Figs. 2 and 4 in Ref. [44]). 

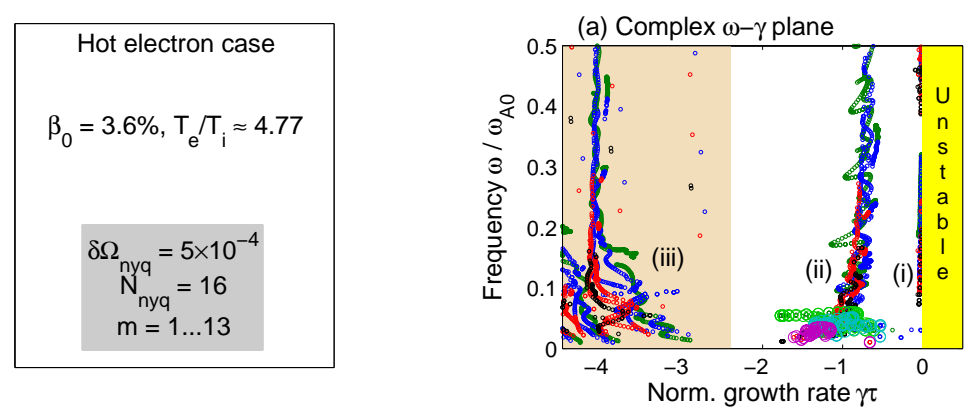

(c) Frequency spectra $\omega(\rho)$ for classes (i), (ii), (iii)
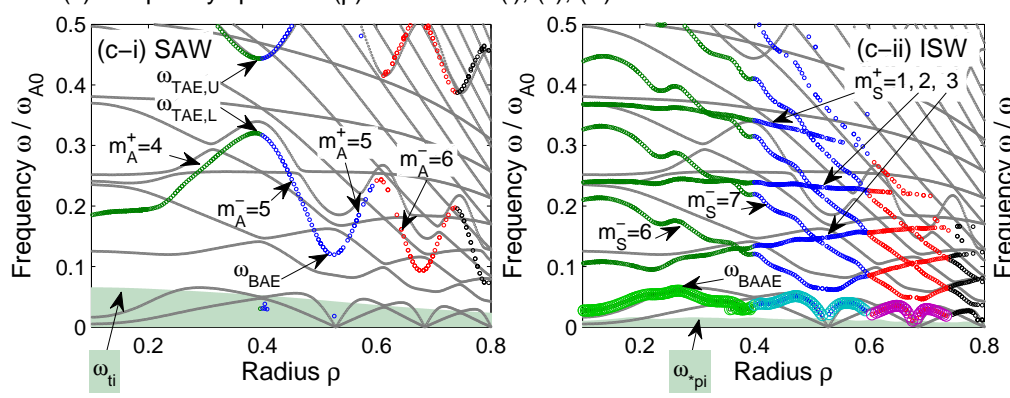
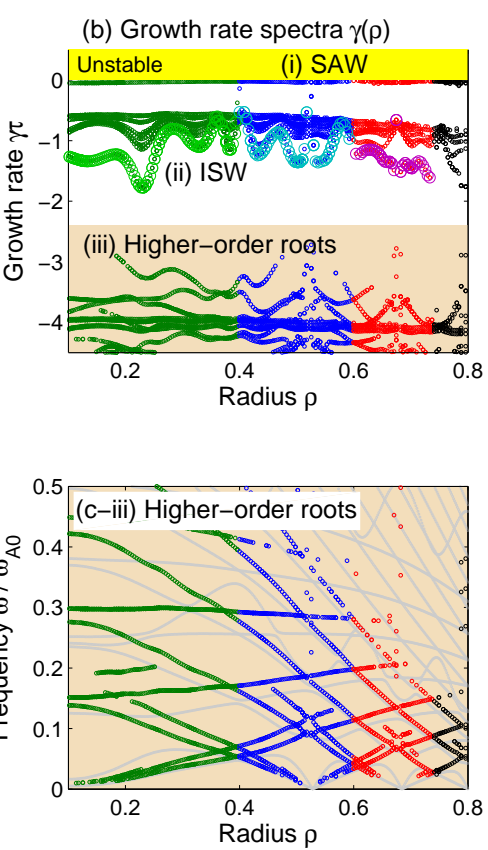

Figure 6. Local analysis results obtained with $T_{\mathrm{i}}=T_{\mathrm{i}}^{\text {ref }}$ and an increased electron temperature $T_{\mathrm{e}}=2.84 \times T_{\mathrm{e}}^{\mathrm{ref}}$, so that $\beta_{0}=3.6 \%$. Arranged as Fig. 5 .

complicated and most likely case-dependent. Here, all ISW continua above the betainduced gap (i.e., far from their respective rational surfaces) are down-shifted by an amount comparable to $\omega_{* \mathrm{pi}}$.

In the following Sections 3.4 and 3.5, we will investigate how the situation changes when the ion and electron temperatures $T_{\mathrm{i}}$ and $T_{\mathrm{e}}$ are varied.

\subsection{Effect of increasing $T_{\mathrm{e}}$ : Reduced damping of ISW}

In the absence of fast ions, the original value of the plasma beta can be recovered if we increase the bulk electron temperature relative to its reference ("ref") value as $T_{\mathrm{e}}=2.84 \times T_{\mathrm{e}}^{\mathrm{ref}}$, which yields $\beta_{0}=\beta_{0}^{\text {ref }}=3.6 \%$ at the axis. With a central value of $T_{\mathrm{e} 0} \approx 6.1 \mathrm{keV} \approx 4.8 \times T_{\mathrm{i} 0}^{\mathrm{ref}}$, the electron temperature is then nearly 5 times greater than the ion temperature. Results for this case are shown in Fig. 6.

Overall, one can see in Fig. 6(c-i) and 6(c-ii) that the SAW (i) and ISW continua (ii) exhibit an increased deviation from the MHD results; in particular, there is a noticeable downshift around $\omega_{\mathrm{TAE}, \mathrm{L}}$ and an even larger downshift around $\omega_{\mathrm{BAE}}$. We have verified that ion diamagnetic effects cause only a negligible shift of the SAW frequencies in the present hot electron case, so we conclude that the large downshifts in Fig. 6(c-i) are mostly due to a change in compressibility.

As expected for an increased temperature ratio $T_{\mathrm{e}} / T_{\mathrm{i}}$, Figs. 6(a) and 6(b) show that the damping rates of the SAW continua (i) in the BAE frequency range $\omega \sim 0.1$ are reduced to $-\gamma \tau \lesssim 0.04$, and the damping of the ISW continua (ii) are reduced to $-\gamma \tau \sim 0.7$ 

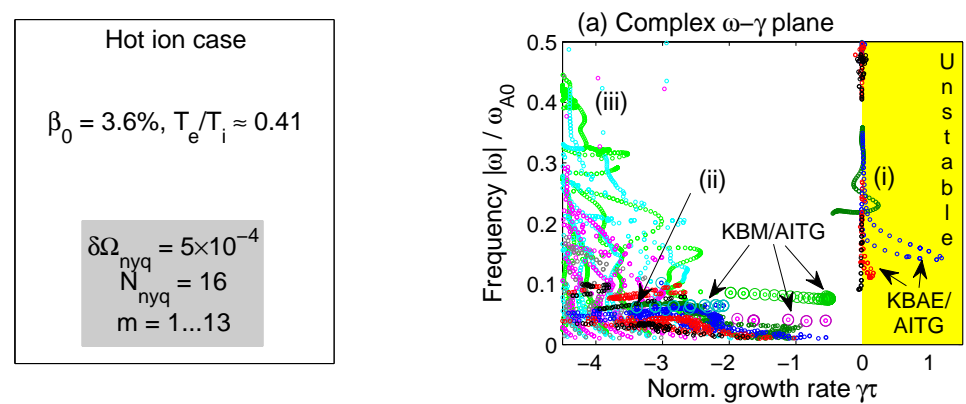

(c) Frequency spectra $\omega(\rho)$ for classes (i), (ii), (iii)
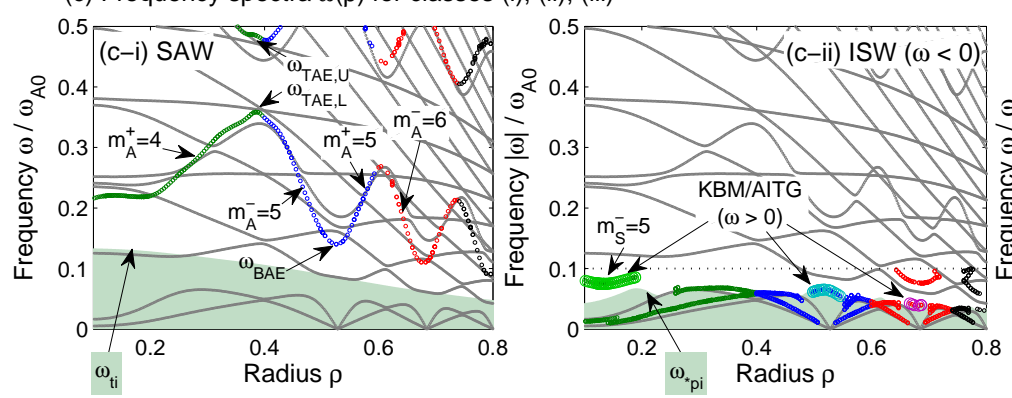

(b) Growth rate spectra $\gamma(\rho)$
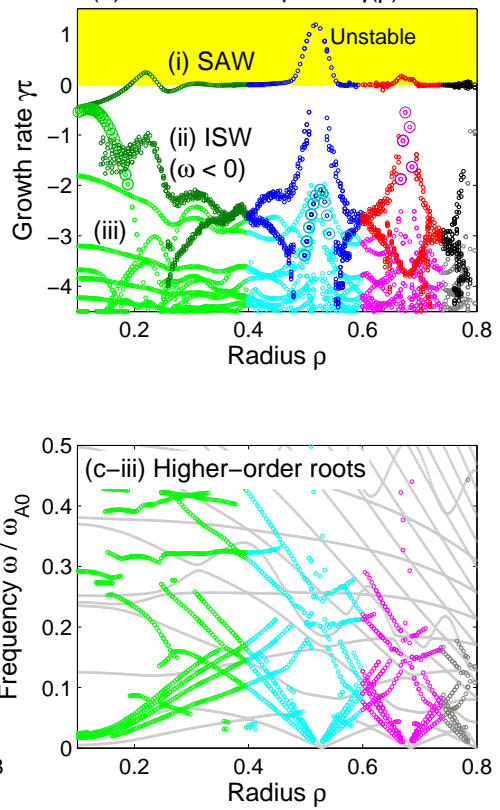

Figure 7. Local analysis results obtained with increased ion temperature $T_{\mathrm{i}}=$ $4.1 \times T_{\mathrm{i}}^{\mathrm{ref}}$ and $T_{\mathrm{e}}=T_{\mathrm{e}}^{\mathrm{ref}}$, so that $\beta_{0}=3.6 \%$. In panels (a) and (c-ii), the absolute value of the real frequency $|\omega|=|\operatorname{Re}\{\Omega\}|$ is plotted in order to show ISW branches with negative frequencies in the range $-0.1 \leq|\omega| \leq 0$ in category (ii). The higher-order roots (iii) are plotted in light colors in order to distinguish them from ISWs with $\omega<0$, which have similar damping rates. Otherwise arranged as Fig. 5.

Note that the wiggles on the ISW continua have become larger due to the higher value of $T_{\mathrm{e}}$, which results in larger variations in $\omega_{* \mathrm{pe}} \propto m\left(n_{\mathrm{e}} T_{\mathrm{e}}\right)^{\prime}$. As mentioned in the previous Section 3.3, these wiggles would be absent if smoother density and temperature profiles were used.

\subsection{Effect of increasing $T_{\mathrm{i}}$ : Destabilization of KBAE/AITG}

Another way to recover the original value of the plasma beta without including fast ions is to increase the bulk ion temperature to $T_{\mathrm{i}}=4.1 \times T_{\mathrm{i}}^{\mathrm{ref}}$, which yields $\beta_{0}=\beta_{0}^{\text {ref }}=3.6 \%$ at the axis. The ion temperature is then more than twice the electron temperature, so that $T_{\mathrm{e} 0} / T_{\mathrm{i} 0} \approx 0.4$ at the axis. The results obtained in this case are shown in Fig. 7.

Overall, one can see in Fig. 7(c-i) that the SAW continua (i) exhibit an increased deviation from the MHD results; in particular, upshifts around $\omega_{\mathrm{TAE}}$ and downshifts around $\omega_{\mathrm{BAE}}$. This can be shown to be due to diamagnetic effects [33].

The KBM/AITG instabilities in the low-frequency domain $\omega \sim \omega_{* \text { pi }}$ of Fig. 7 (c-ii) — which were also seen in the reference case in Fig. 5(c-ii) - are further destabilized. In addition, Figs. 7(a) and 7(b) show that a large part of the SAW continua (i) has become unstable due to the increased ITG drive. These high-frequency AITG instabilities with $\omega \gtrsim \omega_{\mathrm{BAE}}$ are labelled KBAE/AITG in Fig. 7 because we interpret them as the local limit of kinetic BAEs (KBAE) $[44,46]$ driven by ITG. In the present case, the largest 
growth rates are reached near the accumulation points $\omega_{\mathrm{BAE}}$ of the compressibilityinduced gaps. In particular, near the $q=5 / 3$ rational surface at $\rho \approx 0.53$, the local growth rate for roots with $\omega \sim 0.13$ exceeds $\gamma \tau \sim 1$.

Away from the rational surfaces (and from the associated KBM/AITG roots), we do not find any clear trace of ISW branches with positive frequencies $\omega>0$. In contrast to Figs. 5 and 6 above, the ISWs in Fig. 7 are not only absent in the frequency band $0<\omega<\omega_{* \text { pi }}$ (shaded region in Fig. $7(\mathrm{c}-\mathrm{ii})$ ) but became undetectable in the entire positive-frequency plane scanned. However, we were able to find equivalent ISW branches in the negative frequency domain $\omega<0$. The results for the frequency range $-0.1 \leq \omega \leq 0$ are included under category (ii) in Fig. 7 , where the vertical axis of panels (a) and (c-ii) now shows the absolute frequency $|\omega|$. The negative-frequency ISW branches have damping rates that range from relatively low values $-\gamma \tau \sim 1$ near rational surfaces (where KBM/AITG roots are found) to very large values $-\gamma \tau>3$ far away from rational surfaces. It is possible that the positive-frequency ISWs are damped even more strongly, so that they could not be detected with the Nyquist integration contours used.

Interestingly, however, the higher-order roots could still be found in the positivefrequency plane, as can be seen in Fig. 7(c-iii). Moreover, their damping rates shown in Figs. 7(a) and 7(b) have surprisingly decreased and are even lower than in the hot electron case in Fig. 6. This observation remains to be understood, as does the physical meaning of these higher-order roots.

\subsection{Summary and discussion of local analysis results}

The main qualitative discrepancy between MHD and GK solutions is that MHD continua contain many small gaps that are produced by higher-order geometric couplings between SAW and MHD sound branches with $\left|m_{\mathrm{A}}-m_{\mathrm{S}}\right|>1$. Such Alfvén acoustic couplings occur in ideal MHD because all wave branches have purely real frequencies and no damping. In contrast, such higher-order geometric couplings are absent for SAWs and ISWs in the GK description, since SAW tend to be marginally stable and ISW are strongly damped. Only the low-frequency "BAAE gaps" caused by couplings between the lowest-order sidebands $\left|m_{\mathrm{A}}-m_{\mathrm{S}}\right|=1$ remain intact in the GK description.

The largest quantitative discrepancy between MHD and GK solutions was seen in the SAW continua near the BAE accumulation points. In the reference case with $T_{\mathrm{e}} / T_{\mathrm{i}}=1.68$, the $\mathrm{GK}$ value of $\omega_{\mathrm{BAE}}$ was about $20 \%$ below the slow-sound MHD result. That discrepancy increased to 25-30\% when the electron temperature was raised such that $T_{\mathrm{e}} / T_{\mathrm{i}}=4.77$. The discrepancy is even larger for the full-MHD value of $\omega_{\mathrm{BAE}}$. Diamagnetic effects seem to be responsible for only a small part of these discrepancies. For the most part, they can be attributed to differences between the compressibilities in the MHD and GK descriptions. The use of a frequency-dependent specific heat ratio $\Gamma(\omega)$ in MHD may allow to match the GK results more closely.

Concerning the growth/damping rates, increasing $T_{\mathrm{e}}$ was found to reduce the 
damping of both ISWs and SAWs as expected. The effect of increasing $T_{\mathrm{i}}$ was found to depend on the proximity to a rational surface (where $k_{\|} \rightarrow 0$ ). Away from rational surfaces, increasing $T_{\mathrm{i}}$ had a stabilizing effect - so much that for $T_{\mathrm{e}} / T_{\mathrm{i}}=0.41 \mathrm{ISWs}$ with positive frequencies became undetectable in the domain scanned. Near rational surfaces, a strongly destabilizing effect was observed due to the increased ITG drive at least for Alfvénic branches. The destabilized roots were classified as the local limits of KBM/AITG modes in the low-frequency domain $\left(\omega \sim \omega_{* \mathrm{pi}}\right)$ and KBAE/AITG modes in the high-frequency domain $\left(\omega_{\mathrm{BAE}} \lesssim \omega \lesssim \omega_{\mathrm{TAE}}\right)$.

In principle, the effects described above are not new and were seen in one form or another in many previous analytical and numerical studies. The purpose of the present local analysis was to demonstrate and quantify these effects in a particular case with realistic geometry and realistic plasma parameters, and to show the consequences of varying $T_{\mathrm{e}}$ and $T_{\mathrm{i}}$ around their reference values.

We can conclude that continuous spectra obtained with the MHD model may not be sufficiently accurate for the interpretation of modes found in GK simulations (as presented in Section 4 below) or in extended hybrid simulations that include kinetic thermal ion effects (as in Ref. [47]). In particular, for resonantly destabilized Alfvén modes, potentially large discrepancies should be expected in the frequency range $\omega_{\mathrm{BAE}} \lesssim \omega \lesssim \omega_{\mathrm{TAE}}$. This may have an impact on the interpretation of the modes (e.g., gap mode or continuum mode, BAE or KBAE). Moreover, diamagnetic effects are very complex when included self-consistently along with resonant wave-particle interactions in a realistic magnetic geometry, so they cannot be simply accounted for with a mere frequency up-shift as is often done. Caution must also be exercised with high-frequency Alfvén acoustic couplings found in the MHD limit (as in Ref. [1]) whose GK counterparts may not exist or they may exist for different physical reasons.

\section{Global analysis results: Discrete Alfvén eigenmodes and global excitations of ISW continua}

In this section, we study the global plasma response in the frequency range $\omega_{\mathrm{BAE}} \lesssim \omega \lesssim$ $\omega_{\mathrm{TAE}}$. For the interpretation of the results, we shall use the continuous spectra obtained with the GK model in Section 3, since the accuracy of continous spectra obtained from the MHD model is no sufficient for this purpose.

It should be noted that the second-order differential equation used to calculate the local kinetic continua in Section 3 above did not contain higher-order FLR terms that account for effects associated with finite radial wavelengths and are responsible for local electric fields and nonlocal effects such as radiative damping. Meanwhile, these polarization effects are present in the global solutions discussed in the following, where they regularize the otherwise singular solutions near the kinetic continua (e.g., see Fig. 5 in Ref. [21]). In the following, this regularization will be implied when discussing the interactions of the (regularized) kinetic continua with the antenna field or with antennadriven global eigenmodes. Moreover, the radial phase mixing, which is implied by the 


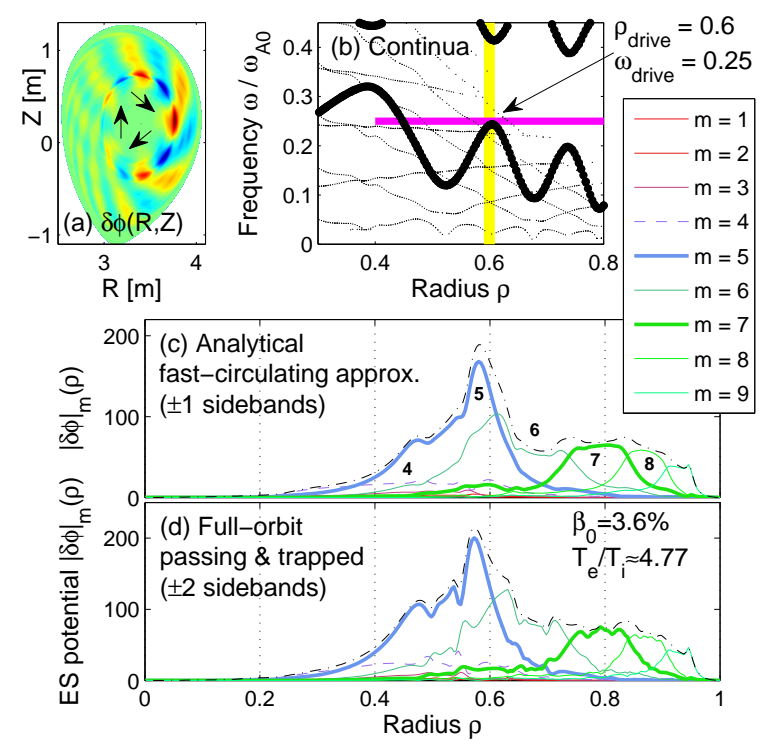

Figure 8. Global structure of an antenna-driven shear Alfvén mode with $n=3$ in the case with increased electron temperature $\left(T_{\mathrm{e}} / T_{\mathrm{i}}=4.77\right)$. Panel (a) shows the structure of the electrostatic (ES) potential fluctuations $\delta \phi(R, Z)$ in the poloidal plane. Panel (b) shows the location $\rho_{\text {drive }}$ and frequency $\omega_{\text {drive }}$ of the antenna in comparison with the GK continua of SAWs (bold) and ISW (thin) obtained with the local analysis in Section 3.4. Panels (c) and (d) show the radial structure of individual poloidal harmonics $|\delta \phi|_{m}(\rho)$ obtained with the analytical and full-orbit versions of LIGKA.

radial dependence of the continuous spectra computed in Section 3, will manifest itself in the following global analysis in the form of continuum damping.

\subsection{Methods}

For the GK analysis of the global plasma response, we use the kinetic antenna model and vector iteration method of LIGKA [15]. We employ the "analytical version" of LIGKA as described in Section 2.3 above, which is sufficient for our purpose. This can be confirmed in Fig. 8, which shows results for an antenna-driven shear Alfvén mode located slightly above the lower accumulation point of the TAE gap formed by the toroidal coupling between $m=5$ and $m=6$. The result obtained with the analytical version and the full-orbit version of LIGKA are plotted in Figs. 8(c) and 8(d), respectively.

One can see that the analytical model reproduces the overall mode structure very well and misses only minor features of the radial sub-structure. Note that the fullorbit version allows the inclusion of higher-order sideband couplings between poloidal harmonics of the quasi-neutrality condition. In Fig. 8(d), couplings between $m, m \pm 1$ and $m \pm 2$ were included, but it is found (not shown here) that the contribution of $m \pm 2$ is negligibly small. Thus, it is sufficient to retain only the lowest-order sideband couplings between $m$ and $m \pm 1$ as in Fig. 8(c).

The antenna will be used to scan the frequency range $0.01 \leq \omega \leq 0.4$ with a step size of $\Delta \omega=10^{-3}$. The results presented here were obtained with $N_{\rho}=256$ radial grid 
points and poloidal harmonics in the range $1 \leq m \leq 9$. In the frequency band and radial domain of interest, no significant changes were seen with higher resolution (512 radial grid points and 13 harmonics).

The amplitudes of the total (volume-integrated) response spectrum will tell us the frequencies of discrete modes that are supported by the plasma. More subtle features will be revealed by examining the response of individual poloidal harmonics in the frequency-radius plane. Note that the presence of the antenna has some influence on the mode structure. Typically, one will see that the poloidal harmonics that dominate near the drive radius $\rho_{\text {drive }}$ are enhanced and distorted by the antenna.

The results of the antenna excitation can be difficult to interpret when there are discrete modes embedded in a continuous spectrum. In such instances, a vector iteration method will be used to single out individual modes. For the KBAE-type modes examined below, it was sufficient to keep only the three dominant poloidal harmonics. The solver converged in less than 20 iterations when started with a guess based on the results of the antenna version.

Both methods yield the complex-valued radial structures of individual poloidal harmonics in terms of the perturbed electrostatic (ES) and magnetic potentials, $\delta \phi_{m}(\rho)$ and $\delta \psi_{m}(\rho)$. The non-ideal (and non-Alfvénic) component associated with kinetic compression is measured by the parallel ES potential defined as

$$
\delta \Phi_{\|} \equiv \delta \phi-\delta \psi=k_{\|} \delta E_{\|},
$$

where $\delta E_{\|}$is the electric field measured along the magnetic field.

The results reported in this paper were obtained with an antenna driving only $\delta \psi$. For modes close to marginal stability (stable or unstable) that have $\delta \phi \approx \delta \psi$, the antenna drive introduces only a relatively small and insignificant amount of spurious $\delta \Phi_{\|}$fluctuations. This can be (and was) verified by comparing the radial structures and damping/growth rates of the antenna-driven modes with the solutions obtained with the vector iteration method. In contrast, for strongly damped modes, a large $\delta \Phi_{\|}$ component is introduced when only $\delta \psi$ (or $\delta \phi)$ is driven, so that the antenna-driven modes differ from solutions obtained with other methods. In this case the antenna modes are not eigenmodes of the system.

The antenna modes can be directly excited on both SAW and ISW continua. However, this means that we will not be able to ascertain whether there are intrinsic couplings between these two branches. Although it is reasonable to assume that such couplings do exist (as will be discussed in Section 5 below), we have to assume that the arbitrary form of the antenna artificially enhances the ratio $\left|\delta \Phi_{\|}\right| /|\delta \phi|$, so that the Alfvén-to-acoustic mode conversion rates will most likely be overestimated.

In principle, it should be possible to design an antenna model with $\delta \phi_{\text {drive }}=\delta \psi_{\text {drive }}$ such that only Alfvénic fluctuations with $\delta \Phi_{\|} \approx 0$ are driven selectively. However, this is rather difficult to implement because the drive would need to have precisely the form of the self-consistent frequency-dependent local solution of the coupled quasineutrality and GK momentum equations and their boundary conditions. Otherwise, it was found 
that excessively large $\delta \Phi_{\|}$fluctuations are produced, which entirely obscure the results.

\subsection{Discrete spectra of Alfvén eigenmodes: TAEs and KBAEs}

Before searching for subtle ISW signals, it is instructive to examine the discrete spectra of Alfvén eigenmodes in the present configuration, which is the purpose of this section. As in the local analysis presented in Section 3 above, we will consider three cases with different temperature ratios; namely, the hot electron case with $T_{\mathrm{e}} / T_{\mathrm{i}}=4.77$, the reference case with $T_{\mathrm{e}} / T_{\mathrm{i}}=1.68$, and a hot ion case with $T_{\mathrm{e}} / T_{\mathrm{i}}=0.51$ (i.e., with $T_{\mathrm{i}}$ and $\beta_{0}$ somewhat smaller than in Section 3 above). The on-axis beta values lie in the range $1.7 \% \lesssim \beta_{0} \lesssim 3.6 \%$. We focus on responses with frequencies above the beta-induced gap, $\omega \gtrsim \omega_{\mathrm{BAE}} \approx 0.1$.

In the hot electron case shown in Fig. 9, we have selected three peaks in the antenna response amplitude. At the peaks labeled (A) and (B), we find modes with a TAE-like structure, where neighboring harmonics $m$ and $m+1$ of the electrostatic (ES) potential $\delta \phi_{m}(\rho)$ have similar amplitudes in the respective gaps. For these modes, the ratio of the parallel and the total ES potentials is $\left|\delta \Phi_{\|}\right| /|\delta \phi| \sim 10 \%$ or less in the region away from the antenna location, which confirms their Alfvénic nature. The third peak labeled $(\mathrm{C})$ is an antenna mode that forms at the point where the external drive resonates with the $m_{\mathrm{A}}^{ \pm}=5$ branch of the (regularized) SAW continuum. In other words, this is not an eigenmode of the system and is strongly influenced by the antenna location $\rho_{\text {drive }}$ as one can see by comparing Figs. 9(b) and 9(c). This externally forced mode has a larger compressional component, with $\left|\delta \Phi_{\|}\right| /|\delta \phi| \sim 25 \%$.

In the reference case, results for which are shown in Fig. 10, the TAEs are more numerous and have higher frequencies due to the lower value of the plasma beta. The TAEs labeled (E) and (F) in Fig. 10 correspond to modes (A) and (B) in Fig. 9. The additional TAE labeled (D) in Fig. 10 has formed in the lower part of the gap associated with the $m=4$ and $m=5$ harmonics and couples to higher harmonics through all the outer gaps without intersecting a SAW continuum. Consequently, mode (D) is subject to very little damping, which is why the associated peak of the antenna response is very sharp in $\omega$. All three TAEs (D)-(F) have a weak compressional component, with $\left|\delta \Phi_{\|}\right| /|\delta \phi| \lesssim 6 \%$ away from the antenna location.

Next, let us consider cases with high ion temperature. The results of the local analysis for $T_{\mathrm{i}} / T_{\mathrm{e}}=0.41$ and $\beta_{0}=3.6 \%$ in Section 3.5 above (Fig. 7) told us that a large part of the SAW continua is destabilized by ITG. Indeed, the global analysis of the hot ion case revealed a set of discrete modes in the frequency range $\omega_{\mathrm{BAE}} \lesssim \omega \lesssim \omega_{\mathrm{TAE}}$, some of which are unstable. The results were found to be sensitive to the ion temperature, which was scanned in the range $2.0 \leq T_{\mathrm{i}} / T_{\mathrm{i}}^{\mathrm{ref}} \leq 6.4$ while fixing $T_{\mathrm{e}}=T_{\mathrm{e}}^{\mathrm{ref}}$. The corresponding temperature ratios were $0.8 \geq T_{\mathrm{e}} / T_{\mathrm{i}} \geq 0.3$. Here, we will only report the results for one selected example, where $T_{\mathrm{e}} / T_{\mathrm{i}}=0.51$ with $T_{\mathrm{i}}=3.3 \times T_{\mathrm{i}}^{\mathrm{ref}}$.

Figure 11 shows the results obtained with the kinetic antenna model. When the antenna is applied at $\rho=0.586$ (i.e., inside the $m=5,6$ TAE gap), the frequency 

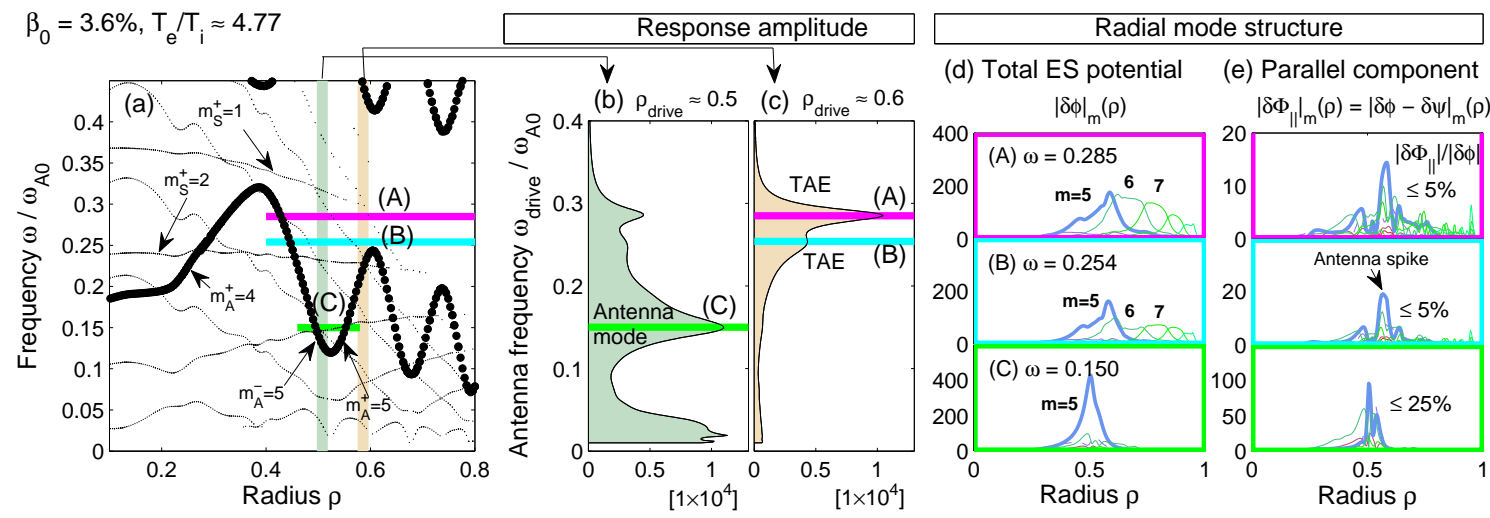

Figure 9. Volume-integrated antenna response amplitude spectra and three discrete global modes $(\mathrm{A})-(\mathrm{C})$ in the case with high electron temperature, $T_{\mathrm{e}} / T_{\mathrm{i}}=4.77$. Panel (a) shows the continuous spectra of SAW and ISW obtained with the local GK analysis (cf. Fig. 6). Two vertical bars indicate the radii $\rho_{\text {drive }}=0.508$ and 0.586 where the antenna is applied. The respective total (volume-integrated) response amplitudes are shown in panels (b) and (c) as functions of the antenna frequency $\omega_{\text {drive. Three of the }}$ peaks seen in the response spectrum are selected and labeled (A), (B) and (C). Their frequencies $\omega_{(\mathrm{A})}=0.285, \omega_{(\mathrm{B})}=0.254$, and $\omega_{(\mathrm{C})}=0.15$ are indicated as horizontal bars in (a)-(c), and their radial structures are shown in columns (d) and (e) in terms of the total electrostatic (ES) potential $|\delta \phi|_{m}(\rho)$ and its parallel (compressional) component $\left|\delta \Phi_{\|}\right|_{m}(\rho)$ defined as $\delta \Phi_{\|} \equiv \delta \phi-\delta \psi=k_{\|} \delta E_{\|}$.
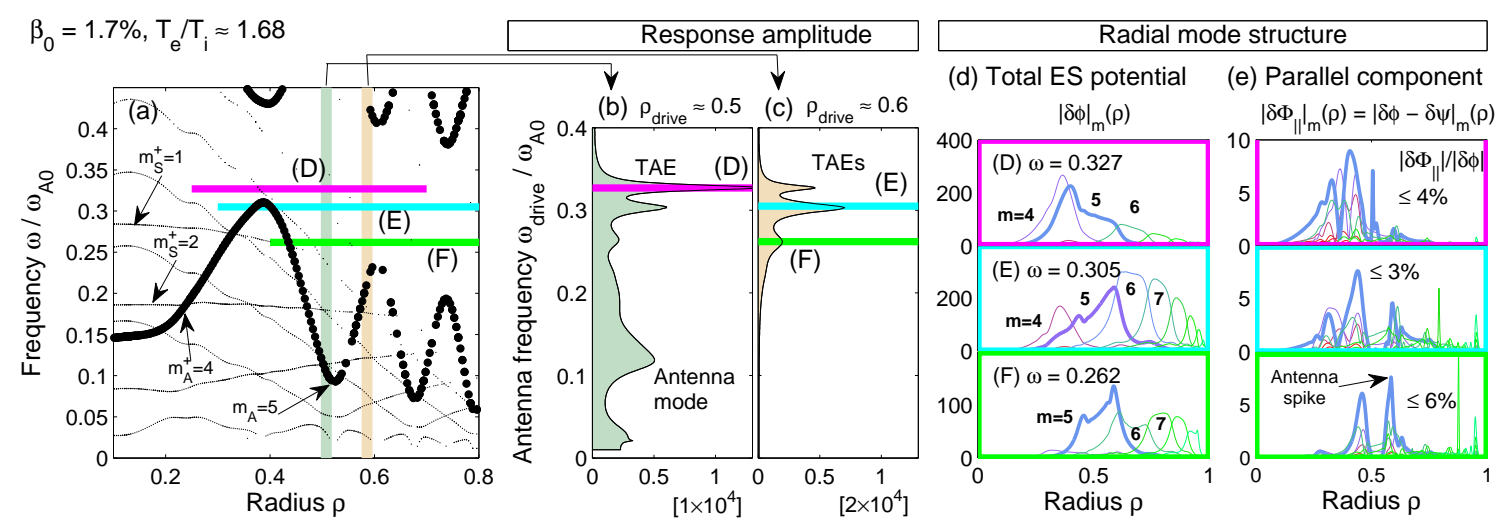

Figure 10. Volume-integrated antenna response amplitudes and discrete global modes (D) $-(\mathrm{F})$ in the reference case with $T_{\mathrm{e}} / T_{\mathrm{i}}=1.68$. Arranged as Fig. 9. The continuous spectra in panel (a) are from Fig. 5 . The selected mode frequencies are $\omega_{(\mathrm{D})}=0.327$, $\omega_{(\mathrm{E})}=0.305$, and $\omega_{(\mathrm{F})}=0.262$.

spectrum of the volume-integrated response amplitude in Fig. 11(c) shows two broad overlapping peaks, which we labeled $(\mathrm{G})$ and $(\mathrm{H})$. Based on their radial structures in Fig. $11(\mathrm{~d})$ and the fact that $\left|\delta \Phi_{\|}\right| /|\delta \phi| \lesssim 5 \%$, these modes are interpreted as TAEs. There was no distinct phase flip in the antenna response, which indicates that these TAEs are strongly driven by the exaggerated ITG.

In addition, a sharp narrow spike labeled (I) can be seen at a lower frequency $\omega=0.152$. This frequency is located above the BAE accumulation point $\omega_{\mathrm{BAE}}(m=$ 
Gyrokinetic analysis of low-n SAW and ISW spectra in a high-beta tokamak plasma 24
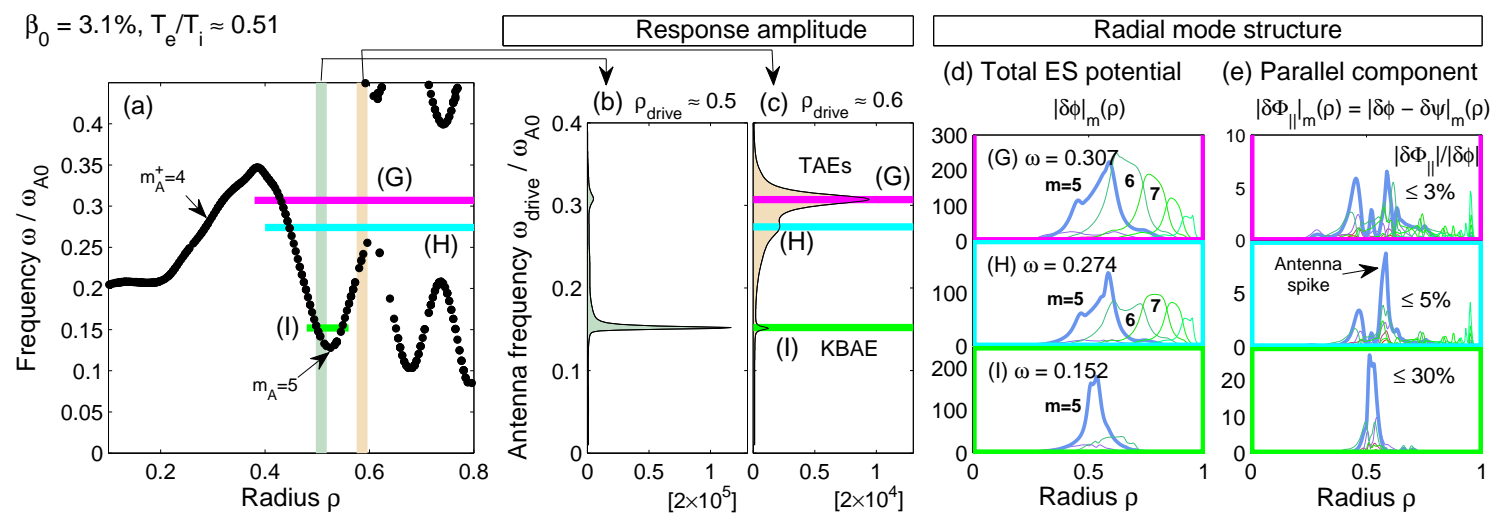

Figure 11. Volume-integrated antenna response amplitudes and discrete global modes (G)-(I) in a case with high ion temperature, where $T_{\mathrm{e}} / T_{\mathrm{i}}=0.51$. Arranged as Fig. 9. The continuous spectra in panel (a) are from Fig. 7. The mode frequencies are $\omega_{(\mathrm{G})}=0.307, \omega_{(\mathrm{H})}=0.274$, and $\omega_{(\mathrm{I})}=0.152$.

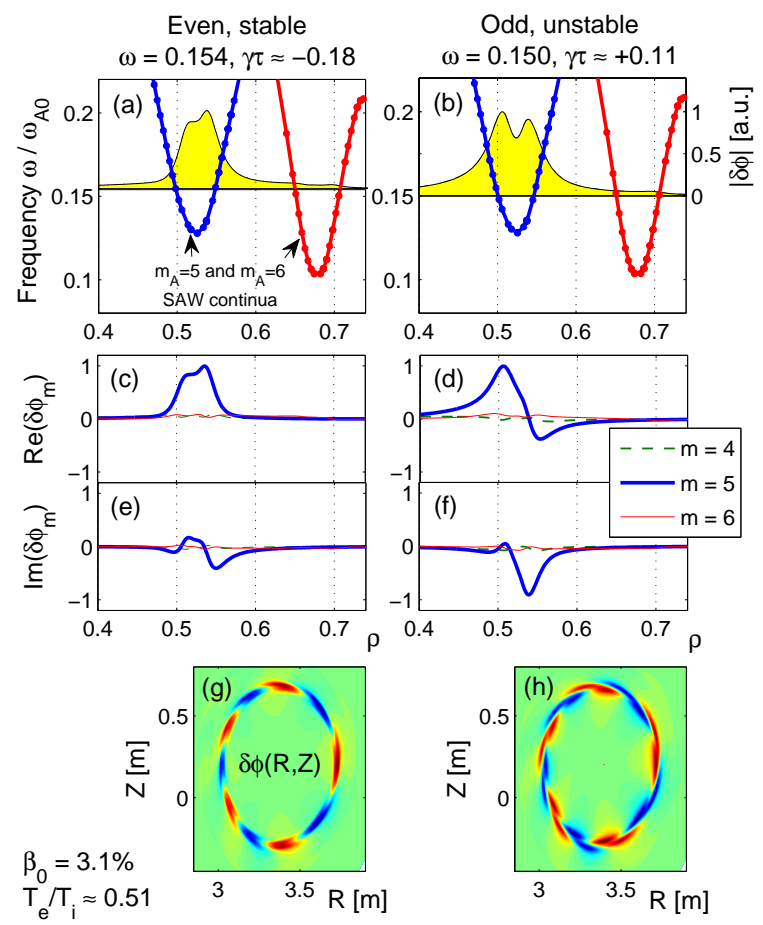

Figure 12. Eigenmodes obtained with the vector iteration solver in the KBAE frequency range for the same hot ion case as in Fig. 11, where $T_{\mathrm{e}} / T_{\mathrm{i}}=0.51$. The left column shows a marginally stable even mode and the right column shows a marginally unstable odd mode. Both have $\left|\delta \Phi_{\|}\right| /|\delta \phi| \lesssim 25 \%$, and only the total ES potential $\delta \phi$ is shown here. (a) and (b): Frequency and radial mode envelope $|\delta \phi|(\rho)$ compared to the SAW continua (c)-(f): Radial structure of the real and imaginary parts of individual poloidal harmonics $\delta \phi_{m}(\rho)$. (g) and (h): Contours of $\delta \phi(R, Z)$ in the poloidal plane. 

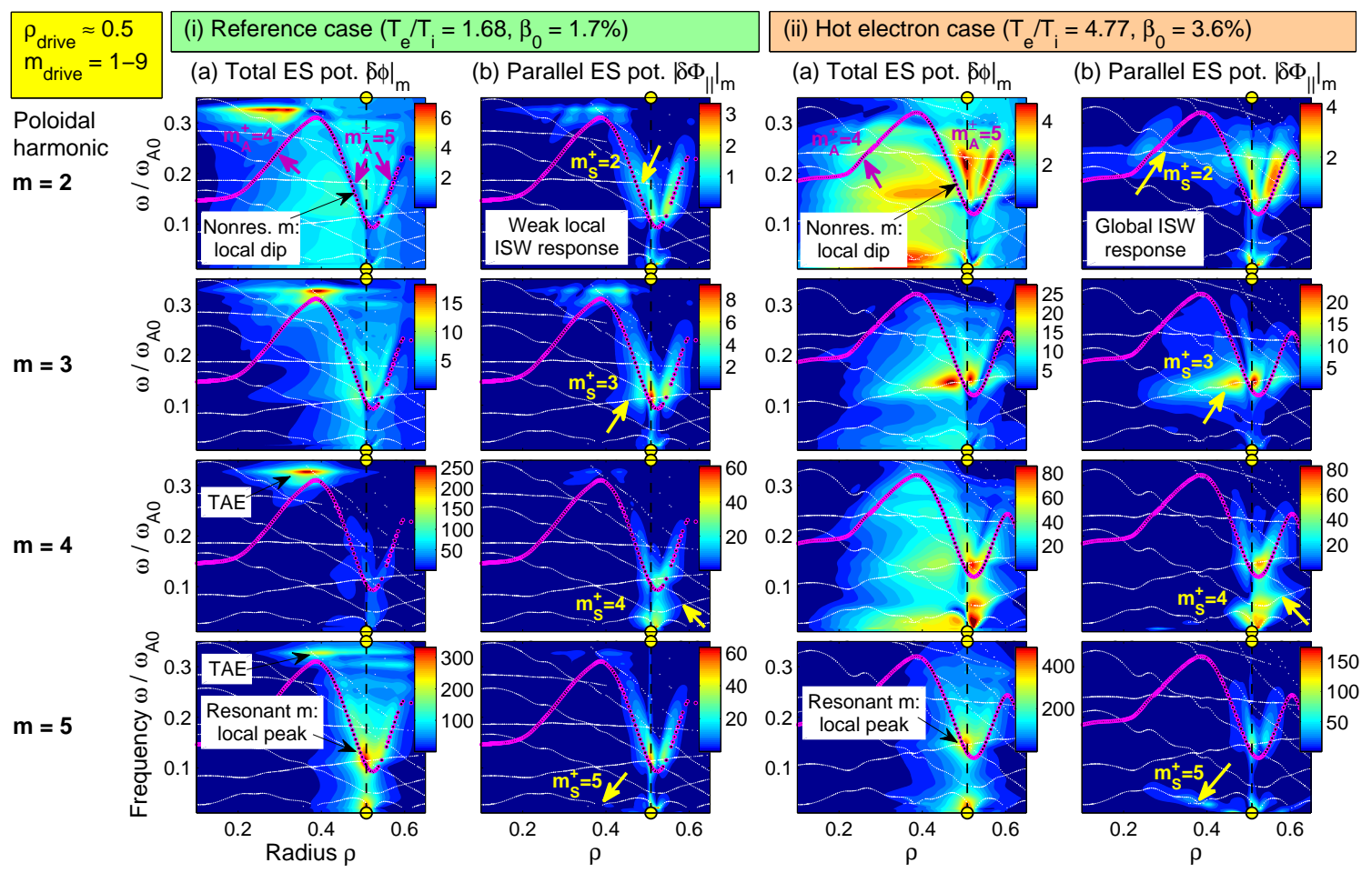

Figure 13. Response amplitude spectra in the frequency-radius plane $(\omega, \rho)$ for the poloidal harmonics $m=2-5$ (from top to bottom). Results are shown for the reference case (i) and the hot electron case (ii). The antenna is located at $\rho=0.508$ (vertical dashed line) and drives $m=1-9$. Columns (a) show the fluctuation spectra of the total ES potential $|\delta \phi|_{m}(\omega, \rho)$ and columns (b) show its parallel component $\left|\delta \Phi_{\|}\right|_{m}(\omega, \rho)$. The contour plots are overlaid with the continua of SAWs (bold) and ISWs (thin), which were obtained with the linear GK analysis in Section 3.

$5) \approx 0.128$. The corresponding mode structure is dominated by $m=5$ and peaks at $\rho \approx 0.5$, between the $m_{\mathrm{A}}^{ \pm}=5$ branches of the SAW continuum as shown in Fig. 11(a), so we interpret this mode as a $\operatorname{KBAE}[44,46]$. Since this mode is present for both $\rho_{\text {drive }}=0.508$ in Fig. $11(\mathrm{~b})$ and $\rho_{\text {drive }}=0.586$ in Fig. $11(\mathrm{c})$, we can be certain that this is not an antenna mode.

Although the amplitude of the antenna response in Figs. 11(b) and 11(c) has a sharp spike at frequency $\omega=0.152$, the phase of the antenna response (not shown here) exhibits a $2 \pi$ jump at a lower frequency $\omega=0.147$. This discrepancy hints at the existence of multiple modes with frequencies near the peak labeled (I) in Fig. 11. Thus motivated, the vector iteration solver was used to search for global eigenmodes with frequencies near $\omega \approx 0.15$. Among many strongly damped solutions, we found two marginally stable/unstable modes as shown in Fig. 12.

The left column of Fig. 12 shows a weakly damped mode with an even parity at $\omega=0.155$, which is similar to the KBAE found with the antenna in Fig. 11(I). The right column of Fig. 12 shows a weakly unstable mode with an odd parity at $\omega=0.150$. Both solutions are dominated by a single poloidal harmonic $m=5$ and are located between the $m_{\mathrm{A}}^{ \pm}=5$ branches of the SAW continuum, so we classify them as KBAEs. 


\subsection{Excitation of continuous spectra: Radial spreading of KAWs and ISWs and possibility of Alfvén acoustic mode conversion}

In this section, we analyze the responses of individual poloidal harmonics in the frequency-radius plane. We begin with a comparison between the reference case $\left(T_{\mathrm{e}} / T_{\mathrm{i}}=1.68, \beta_{0}=1.7 \%\right)$ and the hot electron case $\left(T_{\mathrm{e}} / T_{\mathrm{i}}=4.77, \beta_{0}=3.6 \%\right)$. Figure 13 summarizes the fluctuation spectra for the poloidal harmonics $m=2,3,4$ and 5 when the antenna is located near the $q=5 / 3$ rational surface $\left(\rho_{\text {drive }}=0.508\right)$ and drives all nine harmonics $\left(m_{\text {drive }}=1-9\right)$ that are used in the simulation.

When the fluctuations of the total ES potential $|\delta \phi|_{m}(\omega, \rho)$ in columns (a) of Fig. 13 have significantly larger amplitudes than its parallel component $\left|\delta \Phi_{\|}\right|_{m}(\omega, \rho)$, this is a signature of the discrete Alfvénic modes (or their poloidal sidebands), which were labelled (A)-(C) in Fig. 9 and (D)-(F) in Fig. 10. Apart from these discrete modes, the contour plots of the plasma response in Fig. 13 reveal several other interesting features.

One interesting feature can be seen in the structure of the total ES potential $|\delta \phi|_{m}(\omega, \rho)$ in Fig. 13(a) near the $m_{\mathrm{A}}^{ \pm}=5 \mathrm{SAW}$ continua. While the resonant harmonic $m=5$ has a peak, one can see that the amplitudes of nonresonant harmonics - in particular, $m=2$ and $m=3$ - exhibit a distinct "dip" around the $m_{\mathrm{A}}^{ \pm}=5$ continua. This feature seems to be related to the radial propagation of KAWs, because such dips are only seen when the antenna drives the plasma between two SAW branches with $m_{\mathrm{A}}^{+}=m_{\mathrm{A}}^{-}$, where KAW are reflected back and forth. ${ }^{*}$ We interpret this as a form of kinetic damping of Alfvénic fluctuations, where the SAW continua (here $m_{\mathrm{A}}^{ \pm}=5$ ) convert non-resonant Alfvénic fluctuations (here $m \neq 5$ ) into compressional fluctuations. Through subsequent phase mixing (ion Landau damping) and dissipation, that energy is eventually converted into bulk ion heat.

This conjecture about the occurrence of Alfvén-to-acoustic mode conversion is partly supported by the fact that the parallel ES potential $\left|\delta \Phi_{\|}\right|_{m}(\omega, \rho)$ in Fig. 13(b) shows a relatively strong response just inside the $m_{\mathrm{A}}^{ \pm}=5 \mathrm{SAW}$ continua. Moreover, one may speculate that these compressional fluctuations may excite resonant ISW continua, if they happen to exist in the same region of the frequency-radius plane. This condition is satisfied in the present high-beta scenario, where the $m_{\mathrm{A}}^{ \pm}=5 \mathrm{SAW}$ branches are intersected $\sharp$ by $m_{\mathrm{S}}^{+}=2$ and 3 ISW continua, which are indicated by arrows in Fig. 13(b). Indeed, Fig. 13 shows evidence for such radial spreading as described in the following. $\dagger \dagger$ below.

In the hot electron case (ii) — where ISW damping is weaker $(-\gamma \tau \sim 0.7$ in Fig. 6) and $\beta_{0}=3.6 \%$ is higher - the fluctuation spectrum of $\left|\delta \Phi_{\|}\right|_{m}$ in Fig. 13(ii-b) shows activity that extends over the entire length of the $m_{\mathrm{S}}^{+}=2$ ISW continuum branch. An

* For instance, the dip disappears when the antenna is applied at $\rho_{\text {drive }}=0.39$ as in Fig. 14 below.

$\sharp$ Here, "intersect" refers only to the real frequency-radius plane, since SAW and ISW continua in the GK description have very different damping rates, so that they are well-separated in the complex plane. $\dagger \dagger$ However, it remains speculative that this is caused by Alfvén-to-acoustic mode conversion as described in the previous paragraph because the antenna itself may excite the ISW continua, too, as we will see in Section 4.4 (Fig. 14) 

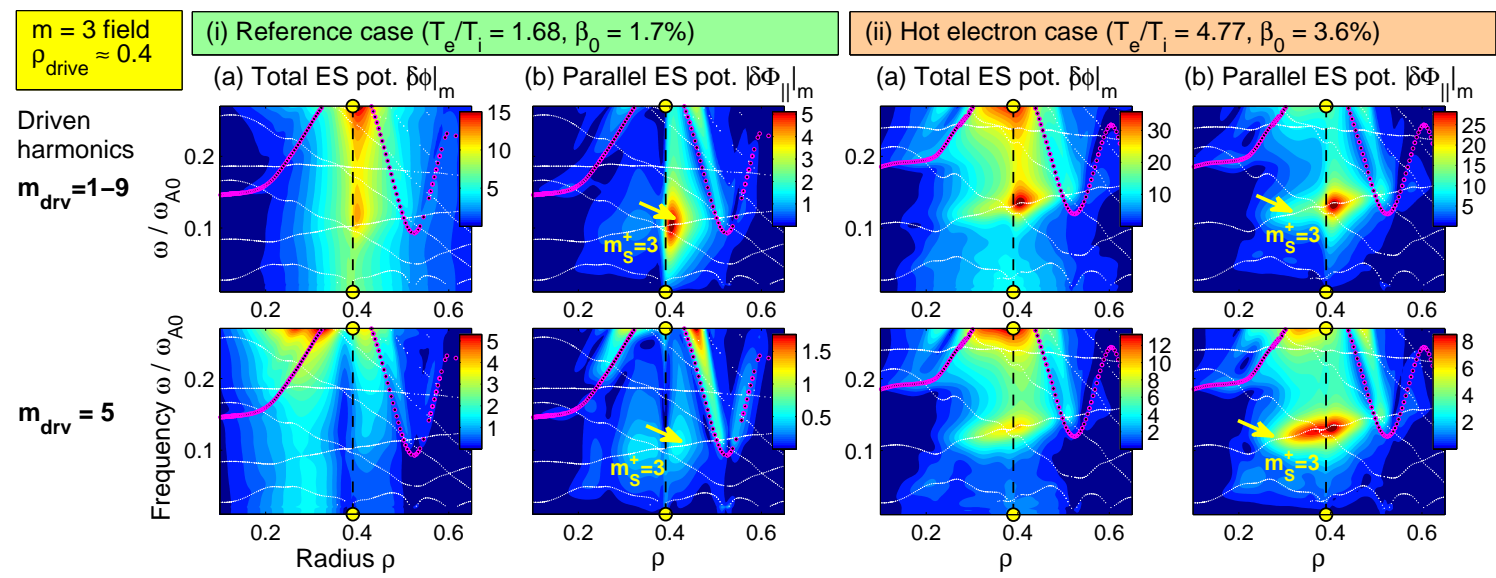

Figure 14. Response amplitude spectra in the frequency-radius plane $(\omega, \rho)$ for $m=3$. The antenna is located at $\rho=0.391$ (vertical dashed line) and drives either all harmonics $m=1-9$ (top) or only $m=5$ (bottom). Otherwise, arranged as Fig. 13.

even stronger compressional response is observed for the $m=3$ harmonic along the $m_{\mathrm{S}}^{+}=3$ ISW branch, which is almost purely electrostatic here, as one can see from the fact that $|\delta \phi|_{m=3} \approx\left|\delta \Phi_{\|}\right|_{m=3}$. The trend of ISW continua with higher $m$ to respond with higher amplitudes can also be seen from the spectra for $m=4$ and $m=5$ in Fig. 13(ii-b), whose ISW continua lie deep in the beta-induced gap.

In contrast, in the reference case (i) — where ISW continua were found to be strongly damped $(-\gamma \tau \sim 2$ in Fig. 5$)$ and the on-axis beta value is only $\beta_{0}=1.7 \%$ - the response of the ISW continua is very localized and weak. In Fig. 13(i-b), only a small amount of radial spreading along the $m_{\mathrm{S}}^{+}=3$ ISW branch can be seen in the $\left|\delta \Phi_{\|}\right|_{m}$ fluctuations of the $m=3$ harmonic. For $m=2$, the ISW continuum response is invisibly small.

Note that the response amplitude of the $m=3$ harmonic in Fig. 13 may also be strongly affected by the antenna, whose location $\rho_{\text {drive }}=0.508$ coincides with the radius where the $m_{\mathrm{S}}^{+}=3$ and $m_{\mathrm{A}}^{-}=5$ continua intersect. Therefore, it is instructive to change the position of the antenna, as we will do next.

\subsection{Excitation of continuous spectra: Role of the antenna's position and mode spectrum}

Figure 14 shows results obtained with the antenna shifted inward to $\rho_{\text {drive }}=0.391$ (near the accumulation point of the $m=4,5$ TAE gap). In addition to the different antenna position, Fig. 14 shows how the plasma response changes when the antenna drives only the $m_{\text {drive }}=5$ harmonic (bottom) instead of all nine harmonics $m_{\text {drive }}=1-9$ (top). Here, we focus on the spectra of the $m=3$ harmonics and compare again the results for the reference case (i) and hot electron case (ii).

The results in Fig. 14 clearly demonstrate that the antenna can directly drive the ISW continua in a region far away from any SAW continuum. Unfortunately, this 
also means that the role of Alfvén-to-acoustic mode conversion for the the excitation of ISW continua as described in Section 4.3 above remains speculative, because the antenna itself may excite the ISW continua directly. We can only say with certainty that Alfvénic and compressional fluctuations are simultaneously excited by the antenna and that they are able to spread radially along the respective continuous spectra.

In addition, Fig. 14 shows that the poloidal mode numbers do not need to match: it is even possible for an antenna with $m_{\text {drive }}=5$ to excite the $m_{\mathrm{S}}^{+}=3$ continuum; although, the response tends to be more diffuse than in the case where the $m_{\text {drive }}=3$ harmonic is part of the antenna field. Note that the amplitude of the $m=3$ ISW fluctuations is about $25 / 5=5$ times larger in the hot electron case (ii) than in the reference case (i) when $m_{\text {drive }}=1-9$ (Fig. 14, top). That ratio increases to about $8 / 0.5=16$ when only the $m_{\text {drive }}=5$ harmonic is driven (Fig. 14, bottom). Clearly, the different values of $T_{\mathrm{e}} / T_{\mathrm{i}}$ and $\beta_{0}$ affect how easily the ISWs can be excited, so we shall now take a closer look at the role of these parameters.

\subsection{Excitation of continuous spectra: Roles of the temperature ratio and plasma beta}

There are two factors that influence how easy or difficult it is to excite ISW, either by direct drive or Alfvén acoustic mode conversion:

- the damping rate of ISWs, and

- the coupling strength between fluctuations with different harmonics or different polarizations.

Of course, these two factors are not completely independent since weaker damping may facilitate stronger coupling or mode conversion. However, the above distinction may be useful since some parameters may affect the coupling strength without changing the damping rates. Candidates that may affect the coupling strength are the value of $\beta$, the poloidal mode number $m$, and the frequency $\omega$. A well-known parameter controlling the ISW damping rate is the temperature ratio $T_{\mathrm{e}} / T_{\mathrm{i}}$.

In order to throw more light on the physics of ISW excitation, Fig. 15 shows the fluctuation spectra of the $m=3$ harmonic of the parallel ES potential $\left|\delta \Phi_{\|}\right|_{m=3} \mid(\omega, \rho)$ for the four cases summarized in Table 1, where we varied the temperature ratio at constant beta and vice versa. The "hot bulk" case (iii) has $T_{\mathrm{e}} / T_{\mathrm{i}}=1.68$ as in the reference case (i), but beta is increased to $\beta_{0}=3.6 \%$ as in the hot electron case (ii) by multiplying both temperatures by 2.155 . The "cold ion" case (iv) has a higher temperature ratio $T_{\mathrm{e}} / T_{\mathrm{i}}=4.77$ as in the hot electron case (ii), but beta is reduced to $\beta_{0}=1.7 \%$ as in the reference case (i) by dividing both temperatures by 2.155. For each case, results are shown for two antenna locations: (a) $\rho_{\text {drive }}=0.391$ and (b) $\rho_{\text {drive }}=0.508$. All nine poloidal harmonics $m=1-9$ are driven.

The results in Fig. 15 show that reducing the ISW damping rate from $-\gamma \tau \sim 2$ to 0.7 by increasing $T_{\mathrm{e}} / T_{\mathrm{i}}$ at constant $\beta_{0}$ causes the ISW amplitudes to increase by a factor of about 1.5-3, depending on the antenna location. In addition, the ISW amplitudes increase by another factor of about 2 when $\beta_{0}$ is doubled at constant $T_{\mathrm{e}} / T_{\mathrm{i}}$. 


\begin{tabular}{l|cc|ccc}
\hline Case & $T_{\mathrm{e}} / T_{\mathrm{e}}^{\mathrm{ref}}$ & $T_{\mathrm{i}} / T_{\mathrm{i}}^{\mathrm{ref}}$ & $T_{\mathrm{e}} / T_{\mathrm{i}}$ & $\beta_{0}$ & $-\gamma \tau$ \\
\hline (i) Reference & 1 & 1 & 1.68 & $1.7 \%$ & 2 \\
(ii) Hot electrons & 2.84 & 1 & 4.77 & $3.6 \%$ & 0.7 \\
(iii) Cold ions & $2.84 / 2.155$ & $1 / 2.155$ & 4.77 & $1.7 \%$ & 0.7 \\
(iv) Hot bulk & 2.155 & 2.155 & 1.68 & $3.6 \%$ & 2 \\
\hline
\end{tabular}

Table 1. Temperature scaling factors, temperature ratios $T_{\mathrm{e}} / T_{\mathrm{i}}$, on-axis beta values $\beta_{0}$ and typical local ISW damping rates $-\gamma \tau$ for cases (i)-(iv) in Fig. 15.

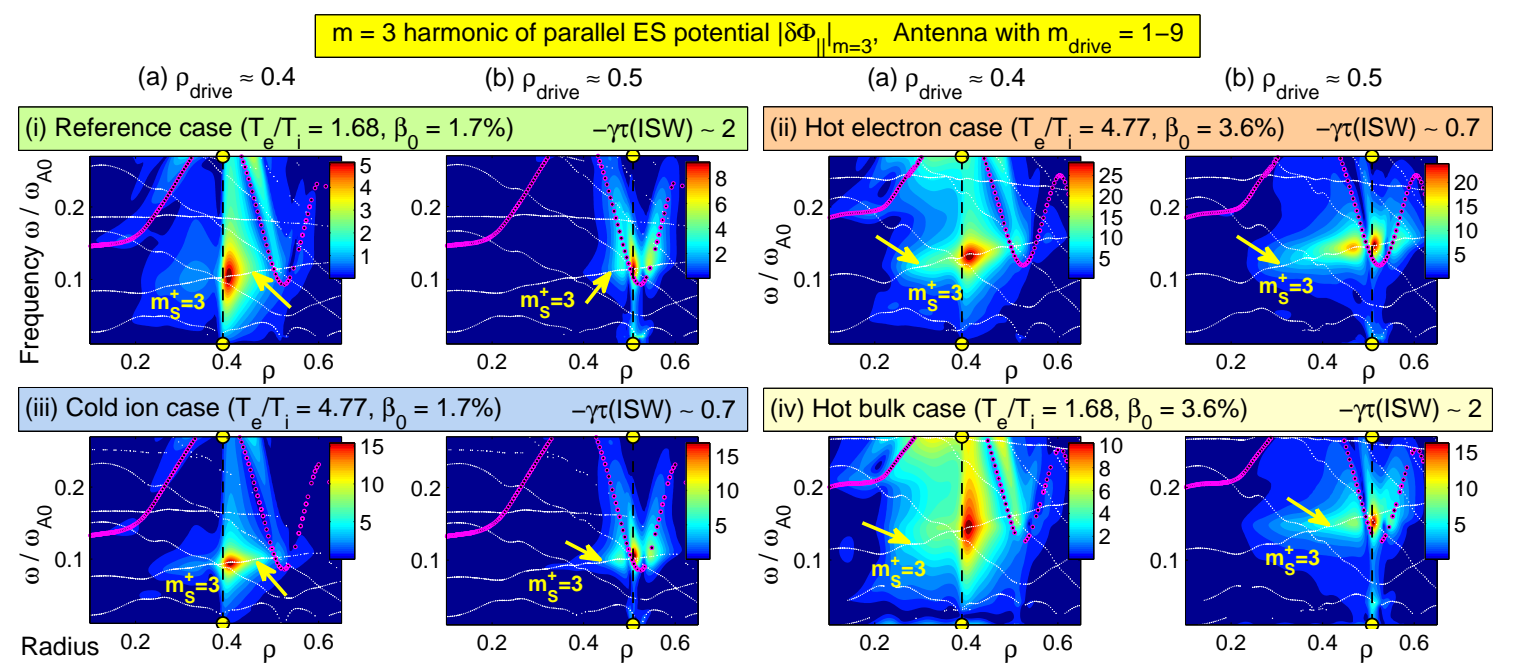

Figure 15. Response amplitude spectra of the parallel ES potential $\left|\delta \Phi_{\|}\right|_{m=3}$ in the frequency-radius plane $(\omega, \rho)$ for the four cases listed in Table 1 , where the temperature ratio is varied at fixed beta and vice versa. The values of the characteristic ISW damping rate $-\gamma \tau(\mathrm{ISW})$ in the local limit are shown for each case. The antenna drives all harmonics $m=1-9$ and is located either at $\rho=0.391$ (a) or $\rho=0.508$ (b).

Furthermore, one can see that a larger $\beta_{0}$ value increases the width of the ISW response along both the frequency axis and the radial axis. One possible explanation is that a higher beta facilitates stronger coupling between different poloidal harmonics, which we already saw in the hot electron case (ii) in Fig. 14 , where $m_{\text {drive }}=5$ was used to excite $m_{\mathrm{S}}^{+}=2$ ISW continua. Alternatively, it is possible that a higher beta enhances the radial propagation of KAWs and/or ISWs.

Although not shown here, we have found that both the cold ion case (iii) and the hot bulk case (iv) exhibit a small but noticeable amount of activity around the $m_{\mathrm{S}}^{+}=2$ ISW continuum, whereas no clear response could be identified in the reference case (i), as we have already pointed out.

The fact that increasing the value of $\beta_{0}$ while fixing $T_{\mathrm{e}} / T_{\mathrm{i}}$ leads to larger ISW amplitudes in spite of constant ISW damping rates confirms our initial expectation that the plasma beta influences the strength of coupling or mode conversion between SAW/KAW and ISW branches. The implications of these results for the possible role of Alfvén acoustic couplings for anomalous bulk ion heating are discussed in the following 
Section 5.

\section{Discussion: Mechanism for Alfvén acoustic coupling in the BAE-TAE frequency range and relevance for anomalous ion heating of bulk ions}

As we have mentioned in our discussion of the results in Section 4.4, it is difficult to claim with certainty that Alfvén acoustic couplings occurred in our antenna simulations, because both branches may be excited by the antenna directly and simultaneously. Nevertheless, it is reasonable to assume that SAWs in a high-beta tokamak plasma always contain some compressional components, so we may conjecture that global shear Alfvén modes will always "couple" to nearby ISW branches and transfer energy to them via a mode conversion process. In other words, it is conjectured that SAW and ISW components of a global mode in a high-beta plasma are intrinsically coupled because they are not perfectly orthogonal functions.

If this is the case, then the amplitude of ISW ("daughter wave") is not determined by the growth/damping rate of the local ISW continuum but by the amplitude of the SAW ("mother wave"). In this way, ISWs with small but possibly significant amplitudes may always be present when there are fast-ion-driven SAWs. Moreover, the speculative Alfvén acoustic couplings may be further enhanced when the mode structure is distorted by FLR and FOW of resonant fast ions. Finally, nearby ISW continua may be excited and cause a radial spreading of the compressional fluctuations as seen in the hot electron case, results for which are summarized once more in Figs. 16(b) and 16(c).

In the present work, fast ions were not included, but the ISW excitation process was simulated using a kinetic antenna model. Based on the results obtained, we assert that the ISWs are excited by a combination of

- geometric sideband couplings, and

- mode conversion between like harmonics $\left(m_{\mathrm{A}}=m_{\mathrm{S}}\right)$.

The process of how an antenna field or resonantly driven Alfvén mode with $m_{\text {drive }}=5$ is thought to drive the $m_{\mathrm{S}}^{+}=3$ ISW branch may be illustrated as follows:

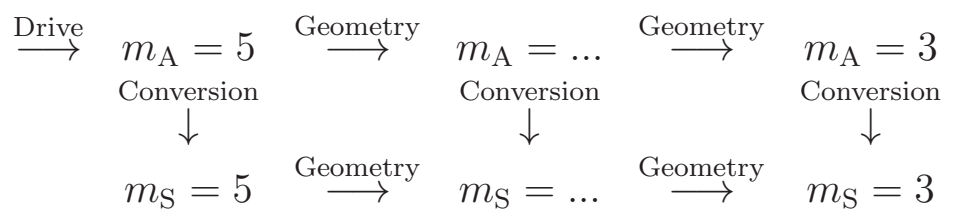

All poloidal harmonics are geometrically coupled by toroidal curvature $(m \pm 1)$ and by the noncircular shape of the plasma cross-section $(m \pm 2,3, \ldots)$. Thus, even when the antenna or the resonant particles drive only the $m_{\mathrm{A}}^{ \pm}=5$ SAW branches (e.g., in the form of a KBAE), the global SAW field will have an $m_{\mathrm{A}}=3$ component. Due to kinetic effects (resonant and nonresonant compression) in a high-beta tokamak plasma, we assume that an Alfvénic fluctuation is always accompanied by some amount of ISWs, as discussed at the beginning of this section. For instance, $m_{\mathrm{S}}=3$ will "piggy-back" on $m_{\mathrm{A}}=3$. There are many such indirect channels to be integrated, and some are shown in 
(a) Channels for anomalous bulk ion heating via SAWs and ISWs

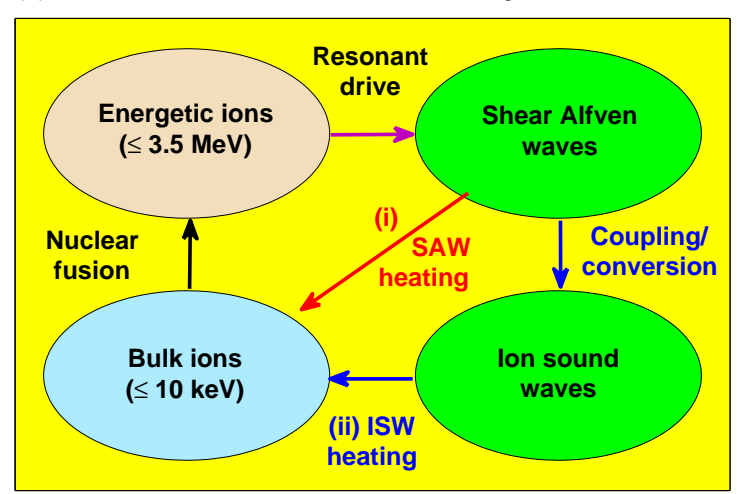

Fluctuation spectra of $\left|\delta \Phi_{\|}\right| \equiv|\delta \phi-\delta \psi|$ for $\mathrm{n}=3$ Hot electron case $\left(\mathrm{T}_{e} / \mathrm{T}_{\mathrm{i}}=4.77, \beta_{0}=3.6 \%, \rho_{\text {drive }}=0.5, \mathrm{~m}_{\text {drive }}=5\right)$

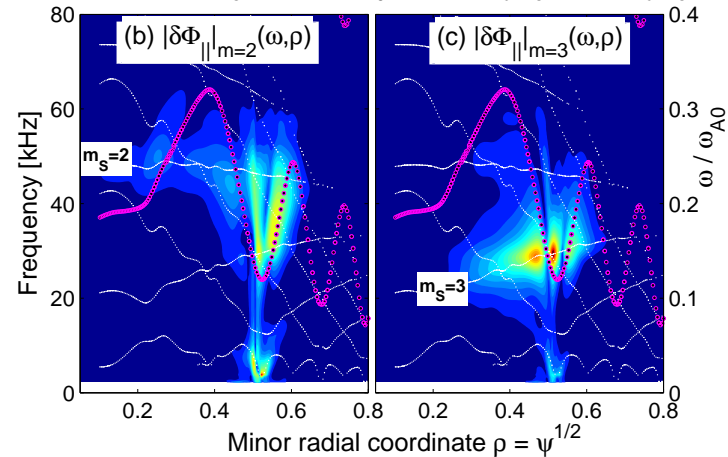

Figure 16. Beta-induced Alfvén continuum modes (BACM) were proposed to provide a new energy channel between fast ions (such as $3.5 \mathrm{MeV}$ alpha particles) and bulk ions via MHD waves as illustrated in panel (a). Panel (b) shows fluctuation spectra of the parallel component of the ES potential $\delta \Phi_{\|}(\omega, \rho)$ for the hot electron case (ii) as in Figs. 13-15 above, but with the antenna driving only $m=5$ at $\rho=0.5$. Results from this case were used to estimate the relevance of ISWs for anomalous heating in Section 5 .

(12) for $m_{\mathrm{A}}=5 \rightarrow m_{\mathrm{S}}=3$. However, due to the large separation between neighboring ISW branches $m_{\mathrm{S}}^{+}$and $m_{\mathrm{S}}^{+} \pm 1$ both in radius and in frequency, it is reasonable to assume that their geometric coupling is negligible, so that we only need to consider the following channel:

$$
\stackrel{\text { Drive }}{\longrightarrow} m_{\mathrm{A}}=5 \stackrel{\text { Geometry }}{\longrightarrow} m_{\mathrm{A}}=3 \stackrel{\text { Conversion }}{\longrightarrow} m_{\mathrm{S}}=3 .
$$

One of the original motivations to study Alfvén acoustic couplings in the BAE-TAE frequency range was that they may contribute to the anomalous heating of bulk ions as illustrated in Fig. 16(a) with the arrow labeled (ii). However, our results show that the ISW tend to have very small amplitudes, so their energy transfer rate should be compared with that of other competing channels. For instance, the kinetic damping of fast-ion-driven Alfvénic fluctuations [48, 49] (which are thought here to give rise to the ISW excitations in the first place) also contributes to anomalous bulk ion heating as illustrated in Fig. 16(a) with the arrow labeled (i). In the following, we estimate the ratio of the energy transfer rates via channels (ii) versus (i) using results from our GK analyses presented in Sections 3 and 4 above.

Using the MHD sound wave dispersion relation $\omega^{2}=k_{\|}^{2} v_{\mathrm{S}}^{2}$, we compute the energies $W_{\mathrm{S}}$ and $W_{\mathrm{A}}$ carried by acoustic and Alfvénic fluctuations with poloidal harmonic $m$ and frequency $\omega$ as follows:

$$
\begin{aligned}
& W_{\mathrm{S}, m}=\left|\delta E_{\|}\right|_{m}^{2}=\left|k_{\|}(\delta \phi-\delta \psi)\right|_{m}^{2}=\frac{\omega_{m}^{2}}{v_{\mathrm{S}}^{2}}|\delta \phi-\delta \psi|_{m}^{2} \\
& W_{\mathrm{A}, m}=\left|\delta E_{\perp}\right|_{m}^{2} \approx\left|\frac{\omega}{k_{\|}} \delta B_{\perp}\right|_{m}^{2}=\left|\delta \psi^{\prime}\right|_{m}^{2}+\frac{m^{2}}{r^{2}}|\delta \psi|_{m}^{2}
\end{aligned}
$$


where $\delta \psi^{\prime}=\partial_{r} \delta \psi$. Letting $\rho \approx r / a$ and noting that $v_{\mathrm{S}}^{2} / v_{\mathrm{A}}^{2}=\Gamma \beta / 2$, we obtain

$$
\frac{W_{\mathrm{S}, m}}{W_{\mathrm{A}, m}}=\frac{2 \varepsilon^{2}}{\Gamma \beta} \frac{\omega_{m}^{2}}{\omega_{\mathrm{A} 0}^{2}} \frac{\left|\delta \Phi_{\|}\right|_{m}^{2}}{\left|\delta \psi^{\prime}\right|_{m}^{2}+\frac{m^{2}}{r^{2}}|\delta \psi|_{m}^{2}} \approx \frac{0.1}{\beta} \frac{\omega_{m}^{2}}{\omega_{\mathrm{A} 0}^{2}} \frac{\left|\delta \Phi_{\|}\right|_{m}^{2}}{\left|\delta \psi^{\prime}\right|_{m}^{2}+\frac{m^{2}}{r^{2}}|\delta \psi|_{m}^{2}} ;
$$

where we have substituted $a / R_{0} \approx 0.3$ and $\Gamma=5 / 3$.

In order to estimate the upper limit for the energy transfer ratio, let us now consider the hot electron case with $T_{\mathrm{e}} / T_{\mathrm{i}}=4.77$ and $\beta_{0}=3.6 \%$, where ISWs reached the largest amplitudes among all the cases that we have studied in this paper. We use a case where the antenna was applied at $\rho=0.5$ and drove only the $m=5$ harmonic. One may think of this procedure as a model for a KBAEs or EPMs with dominant harmonic $(m, n)=(5,3)$ that excites nearby ISW continua with $(m, n)=(2,3)$ and $(3,3)$. The fluctuation spectra $\left|\delta \Phi_{\|}\right|(\omega, \rho)$ for $m=2$ and $m=3$ in this case are shown in Fig. 16 . Substituting the values from the LIGKA simulations into Eq. (16), we obtain

$$
\begin{aligned}
& \frac{W_{\mathrm{S}, m=2}}{W_{\mathrm{A}, m=2}} \sim \frac{0.1}{2 \%} \times 0.24^{2} \times \frac{0.3}{30+15} \approx 2 \times 10^{-4} ; \\
& \frac{W_{\mathrm{S}, m=3}}{W_{\mathrm{A}, m=3}} \sim \frac{0.1}{2 \%} \times 0.14^{2} \times \frac{20}{1400+100} \approx 1 \times 10^{-4}
\end{aligned}
$$

where we have used $\beta(\rho=0.5) \approx 2 \%, \omega_{m=2}=0.24$ and $\omega_{m=3}=0.14$. Next, these two values are multiplied with the ratio $W_{\mathrm{A}, m} / W_{\mathrm{A}}$ of the energy contained in the $m=2$ and $m=3$ sidebands versus the total energy $W_{\mathrm{A}}$ of the antenna-driven Alfvén mode (which is dominated by $m=5$ here). Summation of the results yields

$$
\begin{aligned}
\frac{W_{\mathrm{S}}}{W_{\mathrm{A}}} & =\frac{W_{\mathrm{A}, m=2}}{W_{\mathrm{A}}} \frac{W_{\mathrm{S}, m=2}}{W_{\mathrm{A}, m=2}}+\frac{W_{\mathrm{A}, m=3}}{W_{\mathrm{A}}} \frac{W_{\mathrm{S}, m=3}}{W_{\mathrm{A}, m=3}} \\
& \sim \frac{45}{1 \times 10^{6}} \times 2 \times 10^{-4}+\frac{1500}{8 \times 10^{6}} \times 1 \times 10^{-4}=3 \times 10^{-8} ;
\end{aligned}
$$

Finally, the respective energy transfer rates $\dot{W} \approx \Delta W / \tau=\left(e^{\gamma \tau}-1\right) W$ can be computed using the local damping rates that we found for the SAW and ISW continua in Section 3. Using the values $\gamma_{\mathrm{A}} \tau=-0.03$ and $\gamma_{\mathrm{S}} \tau=-0.7$ from Fig. 6, we obtain

$$
\frac{\dot{W}_{\mathrm{S}}}{\dot{W}_{\mathrm{A}}}=\frac{\left(e^{\gamma_{\mathrm{S}} \tau}-1\right) W_{\mathrm{S}}}{\left(e^{\gamma_{\mathrm{A}} \tau}-1\right) W_{\mathrm{A}}}=\frac{\left(e^{-0.7}-1\right)}{\left(e^{-0.03}-1\right)} \times 3 \times 10^{-8} \lesssim 10^{-6} .
$$

This rough order-of-magnitude estimate suggests that essentially all the energy that fast ions transfer to SAWs in the frequency band $\omega_{\mathrm{BAE}} \lesssim \omega \lesssim \omega_{\mathrm{TAE}}$ is transferred to thermal bulk plasma via the kinetic damping of the SAWs; namely, the SAW heating channel labeled (i) in Fig. 16(a). Only a negligibly small amount of the fluctuation energy can be transferred via the ISW channel (ii) in Fig. 16(a).

This result is mainly a consequence of two factors. One is the small amplitude of the sidebands (here $W_{\mathrm{A}, m=2,3} / W_{\mathrm{A}, m=5} \ll 1$ ) for modes below the TAE gap. The other is the small amount of energy carried by compressional perturbations compared to Alfvénic perturbations, which, in turn, is a consequence of the large radial wave number of Alfvénic modes in the frequency band $\omega_{\mathrm{BAE}} \lesssim \omega \lesssim \omega_{\mathrm{TAE}}$, which gives $\beta^{-1} k_{\|}^{2} /\left(k_{r}^{2}+k_{\vartheta}^{2}\right) \ll 1$. Even if one takes into account effects that produce a smoother 
radial structure (e.g., fast ion FOW) and lets $k_{\perp}^{2} \rightarrow k_{\vartheta}^{2}$, the result in Eq. (20) increases by no more than an order of magnitude to $\dot{W}_{\mathrm{S}} / \dot{W}_{\mathrm{A}} \lesssim 10^{-5}$, which is still insignificantly small. Although increasing the value of $\beta$ may increase the amplitude $\delta \Phi_{\|}$of the compressional component, this should also have little influence on the results, since both quantities enter Eq. (16) as $\left|\delta \Phi_{\|}\right|^{2} / \beta$.

Therefore, we conclude that the Alfvén acoustic self-heating channel proposed in Ref. [1] is negligible for realistic tokamak beta values, even when the temperature ratio is as large as $T_{\mathrm{e}} / T_{\mathrm{i}}=4.8$, which may be possible in burning fusion plasma.

Of course, there are several uncertainties attached to the above estimates. For instance, the local damping rates we used may not be accurate to quantify the decay of global modes. Moreover, the relative amplitudes of SAW and ISW components may be different in the saturated nonlinear regime. However, due to the smallness of the value obtained in Eq. (20), we anticipate that the final conclusion is reliable; namely, that SAW heating channels $[48,49]$ are by far more important than Alfvén acoustic channels.

\section{Summary and conclusions}

Using the linear gyrokinetic code LIGKA, we have studied the structure of the continuous spectra of shear Alfvén waves (SAW) and ion sound waves (ISW) in a highbeta JT-60U tokamak plasma. Particular attention was paid to the frequency band $\omega_{\mathrm{BAE}} \lesssim \omega \lesssim \omega_{\mathrm{TAE}}$, since Alfvén acoustic couplings in that frequency band were recently suggested as a channel for bulk ion heating in high-beta tokamak plasmas [1].

This work was performed in two steps. First, a local analysis was carried out in Section 3, with the purpose to determine how the continuous spectra of the GK model differ from the MHD predictions; in particular, with respect to the ISW branches. Second, using a kinetic antenna model and a vector iteration method, the global plasma response was examined in Section 4 in search for global ISW signals.

The local analysis performed in Section 3 has shown that the structure of ISW continua of the GK model and the MHD sound wave continua of the single-fluid full MHD model are qualitatively similar in the parameter regime $T_{\mathrm{e}} / T_{\mathrm{i}} \gtrsim 1$, which is typical for fusion-relevant plasmas. Quantitative differences were discussed and the possibility of matching the GK results with the MHD model more closely by defining a frequencydependent specific heat ratio $\Gamma(\omega)$ was pointed out.

Using a kinetic antenna model, we were able to excite global fluctuations along ISW continua together with Alfvénic perturbations. A case study has then shown that the ISW response can be enhanced by increasing the values of the plasma beta or the temperature ratio $T_{\mathrm{e}} / T_{\mathrm{i}}$. While the temperature ratio influences the ISW damping rates, the value of beta seems to influence the strength of coupling between the antenna field and the ISWs. Similarly, beta may also enhance Alfvén acoustic couplings, but this mechanism remains speculative because we are not able to isolate it from the antenna's direct influence on the fields.

Based on the simulation results, we have computed in Section 5 an order-of- 
magnitude estimate for the relative energy transfer rate at which an Alfvén acoustic channel as proposed in Ref. [1] may contribute to the anomalous heating of bulk ions. The scenario was set up such as to mimic the excitation of ISWs with poloidal mode numbers $m=2$ and $m=3$ - which intersect $m_{\mathrm{A}}=5$ SAW branches in the real frequency-radius plane — via EPM- or KBAE-like modes with $m=5$ (such as those found in Section 4.2).

It is estimated that the bulk ion heating rate $\dot{W}_{\mathrm{S}}$ due to ISW damping is smaller than the wave-to-particle energy transfer rate $\dot{W}_{\mathrm{A}}$ due to the kinetic damping of SAWs by 6 orders of magnitude. Although several approximations and simplifications were made in the calculation, the smallness of the ratio $\dot{W}_{\mathrm{S}} / \dot{W}_{\mathrm{A}}$ leads us to conclude that Alfvén acoustic channels in the $\omega_{\mathrm{BAE}} \lesssim \omega \lesssim \omega_{\mathrm{TAE}}$ frequency range can be assumed to be insignificant (practically absent) compared to the anomalous bulk heating provided by the kinetic damping of fast-ion-driven SAWs [48, 49].

Note that this conclusion applies only for fluctuations with small toroidal mode numbers $n \gtrsim 1$, such as the $n=3$ harmonic studied in this paper. The situation for high- $n$ modes, where nonlinear mode-mode couplings play an important role, will have to be studied separately.

\section{Acknowledgments}

One of the authors (A. B.) thanks Nobuyuki Aiba, Go Matsunaga and Kouji Shinohara (QST, Japan) for providing MHD equilibrium data and plasma profiles for JT-60U. Moreover, the thanks Kouji Shinohara (QST) for carefully reading the manuscript and offering helpful comments, and Masatoshi Yagi (QST) for continuous encouragement and support of this research.

The work by A.B. has been supported in part by Grants-in-Aid for Scientific Research from the Japan Society for the Promotion of Science (JSPS), Grants No. 25820443 and 16K18341, and by the 2016 Masaji Yoshikawa Prize for Fusion Energy. The work of Ph.L. has been partially carried out within the Enabling Research Project "Nonlinear Energetic Particle Dynamics" (NLED, WP 15-ER-01/ENEA-03) within the framework of the EUROfusion Consortium and has received funding from the Euratom research and training programme 2014-2018 under grant agreement No 633053. The views and opinions expressed herein do not necessarily reflect those of the European Commission.

\section{References}

[1] A. Bierwage, N. Aiba, and K. Shinohara. Alfvén acoustic channel for ion energy in high-beta tokamak plasmas. Phys. Rev. Lett., 114(1):014002, 2015.

[2] M.S. Chu, J.M. Greene, L.L. Lao, A.D. Turnbull, and M.S. Chance. A numerical study of the high- $n$ shear Alfvén spectrum gap and the high- $n$ gap mode. Phys. Fluids B, 4(11):3713, 1992. 
[3] W.W. Heidbrink, E.J. Strait, M.S. Chu, and A.D. Turnbull. Observation of betainduced Alfvén eigenmodes in the DIII-D tokamak. Phys. Rev. Lett., 71:855, 1993.

[4] A.D. Turnbull, E.J. Strait, W.W. Heidbrink, M.S. Chu, H.H. Duong, J.M. Greene, L.L. Lao, T.S. Taylor, and S.J. Thompson. Global Alfvén modes: Theory and experiment. Phys. Fluids B, 5:2546, 1993.

[5] N. Winsor, J.L. Johnson, and J.M. Dawson. Geodesic acoustic waves in hydromagnetic systems. Phys. Fluids, 11(11):2448, 1968.

[6] C.Z. Cheng, L. Chen, and M.S. Chance. High-n ideal and resistive shear Alfvén waves in tokamaks. Ann. Physics, 161:21, 1985.

[7] L. Chen. In Theory of Fusion Plasmas, edited by J. Vaclavik et al (Bologna: SIF), p. 327, 1988.

[8] G.Y. Fu and J.W. Van Dam. Excitation of the toroidicityinduced shear Alfvén eigenmode by fusion alpha particles in an ignited tokamak. Phys. Fluids B, 1(10):1949, 1989.

[9] W. Deng, Z. Lin, I. Holod, Z. Wang, Y. Xiao, and H. Zhang. Linear properties of reversed shear Alfvén eigenmodes in the DIII-D tokamak. Nucl. Fusion, 52(4):043006, 2012.

[10] N.N. Gorelenkov, H.L. Berk, E. Fredrickson, S.E. Sharapov, and JET EFDA Contributors. Predictions and observations of low-shear beta-induced shear Alfvénacoustic eigenmodes in toroidal plasmas. Phys. Lett. A, 370:70, 2007.

[11] N.N. Gorelenkov, H.L. Berk, N.A. Crocker, E.D. Fredrickson, S. Kaye, S. Kubota, H. Park, W. Peebles, S.A. Sabbagh, S.E. Sharapov, D. Stutmat, K. Tritz, F.M. Levinton, H. Yuh, the NSTX Team, and JET EFDA Contributors. Predictions and observations of global beta-induced Alfvénacoustic modes in JET and NSTX. Plasma Phys. Control. Fusion, 49(12):B371, 2007.

[12] N.N. Gorelenkov, M.A. Van Zeeland, H.L. Berk, N.A. Crocker, D. Darrow, E. Fredrickson, G.-Y. Fu, W.W. Heidbrink, J. Menard, and R. Nazikian. Betainduced Alfvén-acoustic eigenmodes in National Spherical Torus Experiment and DIII-D driven by beam ions. Phys. Plasmas, 16(5):056107, 2009.

[13] F. Zonca, L. Chen, and R. A. Santoro. Kinetic theory of low-frequency Alfvén modes in tokamaks. Plasma Phys. Control. Fusion, 38:2011, 1996.

[14] L. Chen and F. Zonca. Theory of Alfvén waves and energetic particle physics in burning plasmas. Nucl. Fusion, 47:S727, 2007.

[15] Ph. Lauber. Super-thermal particles in hot plasmas - Kinetic models, numerical solution strategies, and comparison to tokamak experiments. Phys. Reports, 533:33, 2013.

[16] L. Chen. Theory of magnetohydrodynamic instabilities excited by energetic particles in tokamaks. Phys. Plasmas, 1(5):1519, 1994.

[17] Y. Kusama, G.J. Kramer, H. Kimura, M. Saigusa, T. Ozeki, K. Tobita, T. Oikawa, K. Shinohara, T. Kondoh, and M. Moriyama et al. Characteristics of Alfvén eigenmodes, burst modes and chirping modes in the Alfvén frequency range 
driven by negative ion based neutral beam injection in JT-60U. Nucl. Fusion, 39(11Y):1837, 1999.

[18] Y. Todo, K. Shinohara, M. Takechi, and M. Ishikawa. Computer simulation of frequency sweeping of energetic particle mode in a JT-60U experiment. J. Plasma Fusion Res., 79(11):1107, 2003.

[19] S. Briguglio, G. Fogaccia, G. Vlad, F. Zonca, K. Shinohara, M. Ishikawa, and M. Takechi. Particle simulation of bursting Alfvén modes in JT-60U. Phys. Plasmas, 14(5):055904, 2007.

[20] A. Bierwage, K. Shinohara, Y. Todo, N. Aiba, M. Ishikawa, G. Matsunaga, M. Takechi, and M. Yagi. Self-consistent long-time simulation of chirping and beating energetic particle modes in JT-60U plasmas. Nucl. Fusion, 57(1):016036, 2017.

[21] Ph. Lauber and S. Günter. Damping and drive of low-frequency modes in tokamak plasmas. Nucl. Fusion, 48(8):084002, 2008.

[22] B. Davies. Locating the zeros of an analytic function. J. Comp. Phys., 66:36, 1986.

[23] Ph. Lauber, S. Günter, and S.D. Pinches. Kinetic properties of shear Alfvén eigenmodes in tokamak plasmas. Phys. Plasmas, 12(12):122501, 2005.

[24] A. Bierwage, P. Lauber, N. Aiba, K. Shinohara, and M. Yagi. Alfvén acoustic channel for ion energy in high-beta tokamak plasmas revisited with a linear gyrokinetic model (LIGKA). In Proceedings of the 14th IAEA Technical Meeting on Energetic Particles in Magnetic Confinrment Systems, Sep 1-4, 2015, Vienna, Austria, edited by International Atomic Energy Agency (IAEA), Vienna, 2015. Invited talk I-20.

[25] K. Shinohara, M. Takechi, M. Ishikawa, Y. Kusama, K. Tsuzuki, K. Urata, H. Kawashima, K. Tobita, A. Fukuyama, and C.Z. Cheng et al. Energetic particle physics in JT-60U and JFT-2M. Plasma Phys. Control. Fusion, 46(7):S31, 2004.

[26] A. Bierwage, Y. Todo, N. Aiba, and K. Shinohara. Dynamics of low-n shear Alfvén modes driven by energetic N-NB ions in JT-60U. Nucl. Fusion, 54(10):104001, 2014.

[27] A. Bierwage and K. Shinohara. Orbit-based analysis of nonlinear energetic ion dynamics in tokamaks. I. Effective mode number profile and resonant frequency tracking. Phys. Plasmas, 23(4):042511, 2016.

[28] A. Bierwage and K. Shinohara. Orbit-based analysis of nonlinear energetic ion dynamics in tokamaks. II. Mechanisms for rapid chirping and convective amplification. Phys. Plasmas, 23(4):042512, 2016.

[29] A. Bierwage, Y. Todo, N. Aiba, and K. Shinohara. Sensitivity study for N-NBdriven modes in JT-60U: Boundary, diffusion, gyroaverage, compressibility. Nucl. Fusion, 56(10):106009, 2016.

[30] A. Bierwage, K. Shinohara, Y. Todo, N. Aiba, M. Ishikawa, G. Matsunaga, M. Takechi, M. Toma, and M. Yagi. First-principle simulations reproduce multiple cycles of abrupt large relaxation events in beam-driven JT-60U plasmas. Preprint: 
2016 IAEA Fusion Energy Conference, Kyoto, Japan. Paper TH/4-3. URL: https://nucleus.iaea.org/sites/fusionportal/Pages/Fusion\%20Energy\%20Conference.as

[31] Ph. Lauber, S. Günter, A. Könies, and S.D. Pinches. LIGKA: A linear gyrokinetic code for the description of background kinetic and fast particle effects on the MHD stability in tokamaks. J. Comp. Phys., 226:447, 2007.

[32] S.D. Pinches, L.C. Appel, J. Candy, S.E. Sharapov, H.L. Berk, D. Borba, B.N. Breizman, T.C. Hender, K.I. Hopcraft, G.T.A. Huysmans, and W. Kerner. The HAGIS self-consistent nonlinear wave-particle interaction model. Comp. Phys. Comm., 111(1-3):133, 1998.

[33] Ph. Lauber, M. Brüdgam, D. Curran, V. Igochine, M. Maraschek, K. Sassenberg, S. Günter, M. García-Mu noz, N. Hicks, and the ASDEX Upgrade Team. Kinetic Alfvén eigenmodes at ASDEX Upgrade. Plasma Phys. Control. Fusion, 51(12):124009, 2009.

[34] I. Chavdarovski and F. Zonca. Analytic studies of dispersive properties of shear Alfvén and acoustic wave spectra in tokamaks. Phys. Plasmas, 21(5):052506, 2014.

[35] H.S. Xie. Generalized plasma dispersion function: One-solve-all treatment, visualizations, and application to Landau damping. Phys. Plasmas, 20(9):092125, 2013.

[36] F. Zonca, L. Chen, A. Botrugno, P. Buratti, A. Cardinali, R. Cesario, V.P. Ridolfini, and JET-EFDA contributors. High-frequency fishbones at JET: theoretical interpretation of experimental observations. Nucl. Fusion, 49(8):085009, 2009.

[37] J. Candy. Electron Landau damping of toroidal Alfvén eigenmodes. Plasma Phys. Control. Fusion, 38(6):795, 1996.

[38] L. Chen and A. Hasegawa. Kinetic theory of geomagnetic pulsations: 1. Internal excitations by energetic particles. J. Geophys. Res., 96(A2):1503, 1991.

[39] N. Joiner and A. Hirose. Erratum: "Gyrokinetic verification of the persistence of kinetic ballooning modes in the magnetohydrodynamic second stability regime" [Phys. Plasmas 15, 082107 (2008)]. Phys. Plasmas, 16:069902, 2009.

[40] H. Qin, W. M. Tang, and G. Rewoldt. Linear gyrokinetic theory for kinetic magnetohydrodynamic eigenmodes in tokamak plasmas. Phys. Plasmas, 6(6):2544, 1999.

[41] H.R. Strauss. Nonlinear, three-dimensional magnetohydrodynamics of non circular tokamaks. Phys. Fluids, 19(1):134, 1976.

[42] A. Hasegawa and L. Chen. Plasma heating by Alfvén-wave phase mixing. Phys. Rev. Lett., 32(9):454, 1974.

[43] L. Chen and A. Hasegawa. Plasma heating by spatial resonance of Alfvén waves. Phys. Fluids, 17(7):1399, 1974.

[44] F. Zonca, L. Chen, J. Q. Dong, and R. A. Santoro. Existence of ion temperature gradient shear Alfvén instabilities in tokamaks. Phys. Plasmas, 6:1917, 1999.

[45] J.-B. Girardo, D. Zarzoso, R. Dumont, X. Garbet, Y. Sarazin, and S. Sharapov. Relation between energetic and standard geodesic acoustic modes. Phys. Plasmas, 
21(9):092507, 2014.

[46] X. Wang, F. Zonca, and L. Chen. Theory and simulation of discrete kinetic beta induced Alfvén eigenmode in tokamak plasmas. Plasma Phys. Control. Fusion, 52(11):115005, 2010.

[47] X. Wang, S. Briguglio, L. Chen, C. Di Troia, G. Fogaccia, G. Vlad, and F. Zonca. An extended hybrid magnetohydrodynamics gyrokinetic model for numerical simulation of shear Alfvén waves in burning plasmas. Phys. Plasmas, 18(1):052504, 2011.

[48] A. Hasegawa and L. Chen. Kinetic process of plasma heating due to Alfvén wave excitation. Phys. Rev. Lett., 35(6):370, 1975.

[49] A. Hasegawa and L. Chen. Kinetic processes in plasma heating by resonant mode conversion of Alfvén wave. Phys. Fluids, 19(12):1924, 1976. 